\title{
Collective coordinate approximation to the scattering of solitons in modified NLS and sine-Gordon models
}

\author{
H.E. Baron and W.J. Zakrzewski \\ Department of Mathematical Sciences, Durham University, \\ Durham DH1 3LE, U.K. \\ E-mail: h.e.baron@durham.ac.uk, w.j.zakrzewski@durham.ac.uk
}

ABSTRACT: We investigate the validity of collective coordinate approximations to the scattering of two solitons in several classes of $(1+1)$ dimensional field theory models. We consider models which are deformations of the sine-Gordon (SG) or the nonlinear Schrödinger (NLS) model which posses soliton solutions (which are topological (SG) or non-topological (NLS)). Our deformations preserve their topology (SG), but change their integrability properties, either completely or partially (models become 'quasi-integrable').

As the collective coordinate approximation does not allow for the radiation of energy out of a system we look, in some detail, at how the approximation fares in models which are 'quasi-integrable' and therefore have asymptotically conserved charges (i.e. charges $Q(t)$ for which $Q(t \rightarrow-\infty)=Q(t \rightarrow \infty))$.

We find that our collective coordinate approximation, based on geodesic motion etc, works amazingly well in all cases where it is expected to work. This is true for the physical properties of the solitons and even for their quasi-conserved (or not) charges. The only time the approximation is not very reliable (and even then the qualitative features are reasonable, but some details are not reproduced well) involves the processes when the solitons come very close together (within one width of each other) during their scattering.

KeYwords: Integrable Equations in Physics, Integrable Field Theories

ARXIV EPRINT: 1411.3620v2 


\section{Contents}

1 Introduction 1

2 The two approaches 3

2.1 Collective coordinate approximation 3

2.2 General comments on the numerical approach 4

3 The modified NLS model 5

3.1 The two-soliton configuration for modified NLS 8

3.2 Implementing the approximation in modified NLS 8

$\begin{array}{llr}3.3 & \text { Results for NLS } & 9\end{array}$

$\begin{array}{lll}3.4 & \text { Results for modified NLS } & 13\end{array}$

4 The modified sine-Gordon model $\quad 21$

4.1 The two-soliton configuration for modified sine-Gordon 24

4.2 Implementing the approximation in modified sine-Gordon 24

$\begin{array}{lll}4.3 & \text { Results for sine-Gordon } & 25\end{array}$

4.4 Results for modified sine-Gordon 26

$\begin{array}{llr}5 & \text { Conclusions } & 29\end{array}$

A Expressions for $a_{x}^{(3,-n)}$ and $\alpha^{(3,-n)}$ for $n=0,1,2,3,4 \quad 30$

B Component functions for the effective Lagrangian of the modified NLS 31

\section{Introduction}

Solitons are special solutions of non-linear evolution equations that maintain their shape and energy as they propagate; and when they interact with each other no energy is radiated and a long time after the interaction the only effect is a shift in their positions compared to the positions they would have had if the scattering had not taken place. In $(1+1)$ dimensional models this behaviour is a result of an infinite number of conserved charges constraining the soliton dynamics, and these conserved charges are a consequence of the integrability of the field theory. However, many non-integrable field theories describe processes which are similar to those seen in integrable field theories, for example the scattering of soliton-like structures which do not significantly alter their shape and radiate very little energy during the scattering. These processes prompted the formulation of a concept of quasi-integrability for field theories in $(1+1)$ dimensions in $[1,2]$ and [3]. In these papers modifications of the integrable sine-Gordon and non linear Schrödinger models were analysed and found to possess, sometimes, characteristics similar to their non-perturbed 
counterparts such as an infinite number of asymptotically conserved charges (i.e. charges $Q(t)$ for which $Q(t \rightarrow-\infty)=Q(t \rightarrow \infty))$. The possession of these characteristics is independent of whether the model is topological (like the sine-Gordon model) or non-topological (like the NLS model). However, unlike in the integrable theories, the possession of these characteristics was shown to be dependent on the field configurations and only configurations with additional symmetries demonstrated these integrability-like properties. It was found that these charges are asymptotically conserved in the scattering of two-soliton configurations when the fields are eigenstates of the space-time reflection around a point for some choice of $\left(x_{\Delta}, t_{\Delta}\right)$, given by

$$
P: \quad(\tilde{x}, \tilde{t}) \rightarrow(-\tilde{x},-\tilde{t}), \quad \text { with } \quad \tilde{x}=x-x_{\Delta} \quad \text { and } \tilde{t}=t-t_{\Delta} .
$$

In this paper we compare two approaches to investigating soliton behaviour in different systems; namely, the collective coordinate approximation and the full numerical simulation. The collective coordinate approximation has previously been identified as a useful tool when considering perturbed soliton-bearing equations [4]. Our work is an extension of our previous study in which we compared these two approaches for bright soliton collisions in the integrable NLS model [5]. Here we generalise the method used in [5] to the modified models considered in [2] and [3]; the quasi-integrability of these models allows us to also compare the scattering anomalies (see equation (1.1) of [2]) in addition to the trajectories of the two solitons and this enables us to investigate more thoroughly the extent to which the collective coordinate approximation is useful. For the modified NLS model we are able to return the system to the integrable NLS which we have considered previously by taking our deformation parameter $\epsilon$ equal to zero; this acts as a check of our numerics, particularly in the calculation of the effective Lagrangian which can be computed analytically only in the case $\epsilon=0$.

One may ask why we consider the specific modifications of the Lagrangians as mentioned in [1] for the modification of the NLS model and [3] for the modification of the sine Gordon model. Do these models describe any physical processes or are they studied for other reasons? This was discussed in the above mentioned references, but for completeness, we present some arguments for their choice here too.

The concept of quasi-integrability is still not fully understood and we are still trying to formulate it in well defined mathematical terms. Its origin comes from the observation that in many physical processes (like the scattering of particles at high energies at CERN) the elastic cross sections correspond to large parts $(\sim 20 \%)$ of the total cross sections. This is somewhat surprising given the amount of energy available for the production of particles. On the other hand such particle production in simple mathematical solitonic models is described by radiation (see the Skyrme model) and so, if these two phenomena are connected, the relatively low particle production could be related to the quasi-integrability of the solitonic model.

The papers [1] and [3] have looked at various modifications of the integrable models and related the cases where these models were quasi-integrable to symmetries of solitonic configurations in these models (i.e. considered whether these models allowed these symmetries to hold and whether the initial field configurations possessed them). And, indeed, they found that in models with such symmetries the radiation effects were significantly lower. 
The collective approximation of the solitons, as mentioned before, not only helps to understand physically what is going on in their scatterings but also reduces the required numerical requirements when one tries to study the properties of such scatterings. The method replaces the field equations by equations of a finite dynamical system but at the same time neglects some radiation effects. In [2] we have shown that this approximation (for integrable models) is very good when the solitons are far away from each other but is less reliable if solitons can come close together during the simulation (to roughly within one soliton width of the distance between them). The question then arises whether these properties are still preserved by the modifications of the models and whether the symmetries leading to quasi-integrability of the models still preserve their influence on the behaviour of the solitons when one approximates the soliton dynamics by the dynamics of their collective approximation. Hence in this paper, we look at the modifications of the Lagrangians which were studied in [1] and [3]. As we shall see, the results basically support these expectations. Of course, our results are mainly qualitative (and not very quantitative) as we know that our collective coordinate approximation neglects radiation effects. ${ }^{1}$ This explains our choice of Lagrangians.

This paper is organised as follows: in section 2 we give details of the two approaches we use to investigate the soliton behaviour. In section 3 we describe our modified NLS model and construct an approximation ansatz for a two-soliton field configuration of this model. We then compare the results of the two approaches in this system starting with the $\epsilon=0$ case, when the model returns to the integrable NLS, before discussing the more general $\epsilon \neq 0$ case. In section 4 we describe our modified sine-Gordon model and construct a two-soliton approximation ansatz in this model; we then compare the two approaches in this system. Finally we present our conclusions in section 5 .

\section{The two approaches}

\subsection{Collective coordinate approximation}

The idea of using collective coordinates to describe the main features of the scattering of solitons and other extended structures is quite old. An early work in this area was performed by Thiele [6] who suggested an equation which describes the dynamics of solitons. This was further generalised by Tretiakov and others [7] to a larger system of variables (see also a recent paper [8] which uses such an approach to discuss perturbed NLS equations). In our work we use the approach of Manton [9, 10], which can be used to model the dynamics of solitons in a wide variety of systems and generally reproduces the results of the full simulations in such systems with good accuracy. Any collective coordinate approach reduces an infinite-dimensional problem to a finite dimensional system described by a set of ODEs and so is much quicker to implement than a full numerical simulation. However, the important issue involves choosing the variables that describe, as accurately as possible, the full problem (see for example [11]). The main observation that helps here is the realisation that for a system that possesses free parameters a slow change of these parameters has only

\footnotetext{
${ }^{1}$ So far nobody has found a reliable way of including radiation effects.
} 
a minimal effect on the total energy of the full system and so may be a good approximation to its slow dynamics. Thus one starts with a static solution $\psi\left(x, q_{1}, \ldots, q_{n}\right)$. The energy of this solution is independent of the constant values of the parameters of this solution and changing the field configuration can only increase the energy, so in the field space there are low energy valleys in the direction of the parameters of the solution. Consider now moving solitons. For small velocities of the solitons the motion is easiest along the valleys described by the parameters of the static solution (as then the increase of the energy is only due to the kinetic energy associated with this change which for very slow changes is very small). Thus, it makes sense to approximate the dynamics of slow moving solitons by allowing these parameters to vary in time, i.e. $q_{i}=q_{i}(t)$, and assume that these parameters describe most of the solitons' dynamics.

However, such an approximation neglects other modifications of the fields and, in particular, all radiative corrections to the solitons and so is valid only for very slow motions and when such corrections are small. In practice, we want to use this approximation not only for infinitesimally small velocities and so we include some extra parameters and then check whether their inclusion improves the approximation. Here we are often helped by the physics of the problem, and in integrable models we are sometimes guided by considerations of their conserved quantities.

To describe the dynamics of the collective coordinates we proceed as follows [9]. We start with an approximation ansatz whose form is based on the stationary solution with a suitable choice of parameters, these are then taken as collective coordinates and are allowed to depend on time. These coordinates generally describe physical properties of the soliton such as position, height, etc. This ansatz is then substituted into the Lagrangian density of the system to obtain a coupled system of ODEs for the coordinates. Solving these ODEs describes the time evolution of the coordinates, which in turn tells us how the field evolves in time. In some cases, and sometimes with further simplifying assumptions, the equations of motion for the collective coordinates can be solved analytically; such is the case in [13]. In our work the equations of motion need to be solved numerically and for this we use a 4th order Runge-Kutta method.

\subsection{General comments on the numerical approach}

The work we describe in this paper involves several different numerical techniques. They include performing 'full numerical simulations' and the calculations involving collective coordinates. The two approaches are then compared to each other to assess the validity of the collective coordinate approximations. Both approaches use the 4th order Runge-Kutta method of simulating the time evolution, and we use this method both for the modified NLS equation and for the modified sine-Gordon models.

For the full simulations the implementations are different in these two classes of models as the NLS equation involves only first derivatives with respect to time and the phase of the complex field $\psi$ perform fast rotations with the increase of time, while the modified sine-Gordon model involves only a nonlinear wave motion.

Thus, in the NLS case we choose to perform the simulation in a rotating frame (i.e. we go to the frame in which the phase rotation involves only the additional dynamical variation 
relative to this global rotation). The global rotation is calculated at each value of time and the equation is transformed to that frame. Therefore, in this frame, the further phase variation is small and it is only due to the dynamics of the system of solitons. Consequently, for a given time and position steps of the program, the changes of the derivatives of $\psi$ are kept small.

Our approach is a standard procedure for such fields and more discussion of its use in the NLS case can be found in [2] where it is shown that it has worked very well in this case. The numerical errors are negligible and the results of our simulations are essentially the same when we tested the method by varying a little the parameters of our numerical approach. To obtain reliable simulations we experiment by using various lattice sizes, various numbers of points etc. until we are satisfied that we can 'trust' our results; i.e. when the numerical errors are very small and so are insignificant.

Then we perform many simulations as described in this paper. In fact, most of the results we are presenting here have been obtained on a lattice involving $N=5001$ grid points with lattice spacing $d x=0.01$. As in [2] the initial configurations involve two one soliton fields, with solitons placed at $x= \pm x_{0}$ (as discussed in the text below) and with the fields tied together at $x=0$. Luckily, at small values of $x$ the values of the fields are very close to each other and so the numerical errors due to this joining procedure are negligible (in fact we even smooth the fields there over 3 lattice points).

For our calculations, as the equations are first order in time derivative, we have to take a small time step. We have varied this too and found that we can trust our results when $d t=0.00002$ or smaller. Most of our results that we report in this paper are obtained with this value of $d t$, giving $\frac{d t}{d x^{2}}=0.2$ (as $\frac{d t}{d x^{2}}<1$ we are satisfied that the solution is stable).

In the modified sine-Gordon model the equations are of the wave type and so the numerical calculations are simpler than in the NLS case discussed above. We use the fixed boundary conditions with $N=10001$ points, with the lattice spacing $d x=0.01$ and $d t=0.0001$ (and so $\frac{d t}{d x}=0.01$ ). We absorb the energy at the boundaries but, in fact, very little energy ever reaches the boundaries as the scatterings are very elastic.

In the collective coordinate approximation the ODEs are solved numerically with lattice spacing $d x=0.01$ and $d t=0.005$, and so $\frac{d t}{d x}=0.5$, (the simulations were also run with various values of $d x, d t$ and we are satisfied with the accuracy of the results using the values mentioned above). In addition to this we are required to integrate numerically over $x$ in order to obtain the effective Lagrangian from the Lagrangian density. We compare our numerical integrations with the integrations performed analytically (which can only be achieved when the models are integrable, i.e. when $\epsilon=0$ ) and find the results to be sufficiently accurate for our chosen value of $d x=0.01$.

\section{The modified NLS model}

Here we consider the Lagrangian for a non-relativistic complex scalar field in $(1+1)$ dimensions

$$
\mathcal{L}=\int d x \frac{i}{2}\left(\psi^{*} \partial_{t} \psi-\psi \partial_{t} \psi^{*}\right)-\partial_{x} \psi^{*} \partial_{x} \psi-V\left(|\psi|^{2}\right) \text {. }
$$


This Lagrangian has an internal symmetry $\psi \rightarrow e^{i \alpha} \psi$ for $\alpha \equiv$ constant. The equations of motion are

$$
i \partial_{t} \psi=-\partial_{x}^{2} \psi+\frac{\delta V}{\delta|\psi|^{2}} \psi
$$

together with its complex conjugate. With the NLS potential, $V=V_{\mathrm{NLS}}=\eta|\psi|^{4}$, this becomes the NLS equation and the sign of the parameter $\eta$ determines whether there are bright $(\eta<0)$ or dark $(\eta>0)$ soliton solutions.

The equation (3.2) (with any choice of potential which retains the $\eta$ parameter) admits an anomalous zero curvature representation with the connection given by

$$
\begin{aligned}
A_{x} & =-i T_{3}^{1}+\sqrt{|\eta|} \psi^{*} T_{+}^{0}+\sqrt{|\eta|} \psi T_{-}^{0} \\
A_{t} & =i T_{3}^{2}+i \frac{\delta V}{\delta|\psi|^{2}} T_{3}^{0}-\sqrt{|\eta|}\left(\psi^{*} T_{+}^{1}+\psi T_{-}^{1}\right)-i \sqrt{|\eta|}\left(\partial_{x} \psi^{*} T_{+}^{0}-\partial_{x} \psi T_{-}^{0}\right),
\end{aligned}
$$

where $T_{i}^{n}, i=3,+,-$ and $n$ integer, are generators which satisfy the $\mathrm{SL}(2)$ loop algebra commutation relations (for more results see [2]) and can be realised in terms of the finite $\mathrm{SL}(2)$ algebra generators as $T_{i}^{n} \equiv \lambda^{n} T_{i}$, where $\lambda$ is an arbitrary complex parameter. The curvature of this connection is

$$
\begin{aligned}
\partial_{t} A_{x}-\partial_{x} A_{t}+\left[A_{x}, A_{t}\right]= & X T_{3}^{0}+i \sqrt{|\eta|}\left(-i \partial_{t} \psi^{*}+\partial_{x}^{2} \psi^{*}-\psi^{*} \frac{\delta V}{\delta|\psi|^{2}}\right) T_{+}^{0} \\
& -i \sqrt{|\eta|}\left(i \partial_{t} \psi+\partial_{x}^{2} \psi-\psi \frac{\delta V}{\delta|\psi|^{2}}\right) T_{-}^{0}
\end{aligned}
$$

where $X$ is the anomaly given by

$$
X \equiv-i \partial_{x}\left(\frac{\delta V}{\delta|\psi|^{2}}-2 \eta|\psi|^{2}\right) .
$$

This curvature simplifies to $\partial_{t} A_{x}-\partial_{x} A_{t}+\left[A_{x}, A_{t}\right]=X T_{3}^{0}$ when the equations of motion (3.2) are imposed. For the NLS potential, $V_{\mathrm{NLS}}=\eta|\psi|^{4}$, the anomaly $X$ vanishes and it is this vanishing of the curvature which makes the theory integrable. For a general potential we can carry out the abelianization technique of the integrable field theories, for full details see [2], gauge transforming the connection such that the curvature (3.4) becomes

$$
\partial_{t} a_{x}^{(3,-n)}-\partial_{x} a_{t}^{(3,-n)}=X \alpha^{(3,-n)} ; \quad n=0,1,2, \ldots
$$

Explicit expressions for the first few components of $a_{x}^{(3,-n)}$ and $\alpha^{(3,-n)}$ are given in appendix A. In the example that we consider here it is easy to check that $a_{t}^{(3,-n)}$ satisfies the boundary condition $a_{t}^{(3,-n)}(x=\infty)=a_{t}^{(3,-n)}(x=-\infty)$ and so from (3.6) we have an infinite number of anomalous conservation laws:

$$
\frac{d Q^{(n)}}{d t}=\beta_{n} ; \quad \text { with } \quad Q^{(n)}=\int_{-\infty}^{\infty} d x a_{x}^{(3,-n)} ; \quad \text { where } \quad \beta_{n}=\int_{-\infty}^{\infty} d x X \alpha^{(3,-n)}
$$

for $n=0,1,2, \ldots$ It is clear that when the potential corresponds to the NLS potential, i.e. $V_{\mathrm{NLS}}=\eta|\psi|^{4}$, the anomaly $X$ given in (3.5) vanishes and so does $\beta_{n}$. Therefore the theory with the potential $V_{\mathrm{NLS}}$ is integrable as it has an infinite number of conserved charges $Q^{(n)}$. 
In our modified model we use a perturbation of the NLS potential as in [2]

$$
V=\frac{2}{2+\epsilon} \eta\left(|\psi|^{2}\right)^{2+\epsilon}
$$

so that it returns to the unperturbed NLS potential in the case $\epsilon=0$.

As shown in [2], for $\eta<0$, this model has a one-soliton solution given by

$$
\Psi=\left(\sqrt{\frac{2+\epsilon}{2|\eta|}} \frac{b}{\cosh \left[(1+\epsilon) b\left(x-v t-x_{0}\right)\right]}\right)^{\frac{1}{1+\epsilon}} e^{i\left[\left(b^{2}-\frac{v^{2}}{4}\right) t+\frac{v}{2} x\right]+i \phi},
$$

where $b, \phi, v$ and $x_{0}$ are real parameters of the solution.

For two interacting solitons, as argued in [3], we can take

$$
\psi=\psi_{1}+\psi_{2},
$$

where $\psi_{1}=\Psi\left(x, x_{0}, v, \phi_{1}\right)$ and $\psi_{2}=\Psi\left(x,-x_{0},-v, \phi_{2}\right)$. Such fields describe well two interacting solitons (each at $\pm x_{0}$ with velocity $\pm v$ and their phase difference of $\left(\phi_{1}-\phi_{2}\right)$ ) when they are far apart as then, for any point in $\mathrm{x}$, there is a significant contribution from (at most) one $\psi_{i}$ due to the localised nature of the one soliton solution. Such a field configuration was successfully used in [3] as an initial configuration for the numerical investigations of two soliton scatterings and so we use it here too.

In [3] it was also pointed out that to investigate the anomalies it is more convenient to rewrite $\psi$ in terms of new fields $R$ and $\varphi$ as

$$
\psi \equiv \sqrt{R} e^{i \frac{\varphi}{2}}
$$

Then it was shown there that the field $\psi$ of (3.10) transforms under the parity defined in (1.1) as

$$
P: \quad R \rightarrow R ; \quad \varphi \rightarrow-\varphi+\text { constant. }
$$

and so $X$ is even under $\mathrm{P}$ and $\alpha^{(3,-n)}$ is odd under $\mathrm{P}$ (see also appendix A). But for field configurations which transform as in (3.12) we have

$$
\int_{-\tilde{t}_{0}}^{\tilde{t}_{0}} d t \int_{-\tilde{x}_{0}}^{\tilde{x}_{0}} d x X \alpha^{(3,-n)}=0
$$

where $\tilde{t}_{0}$ and $\tilde{x}_{0}$ are given fixed values of the shifted time and space coordinates introduced in (1.1).

Note that (3.7) shows that $Q^{(n)}\left(t_{2}\right)=Q^{(n)}\left(t_{1}\right)+\int_{t_{1}}^{t_{2}} \beta_{n}\left(t^{\prime}\right) d t^{\prime}$ where we have already taken $\tilde{x}_{0} \rightarrow \infty$. Taking $t_{1}$ and $t_{2}$ appropriately we find that we have non-conserved charges (3.7) that vary in time but are symmetric with respect to $t=t_{\Delta}$. Taking further $\tilde{t}_{0} \rightarrow \infty$ we find that the system has an infinite number of asymptotically conserved charges, i.e.

$$
Q^{(n)}(t=+\infty)=Q^{(n)}(t=-\infty)
$$

This all assumes that the symmetry, which was shown to hold for the initial configuration (3.10) holds at all times but the studies in [3] did show that the initial approximation is very good at all times and the charges are asymptotically conserved. Of course, the question then still arises whether this is also true in the collective coordinate approximation. This is what we discuss in the next section. 


\subsection{The two-soliton configuration for modified NLS}

Here we construct a set of collective coordinates for the study of the scattering of two solitons with $\eta=-1$ in the NLS system with our modified potential. Guided by the ideas of [3] we use a natural extension of our approximation ansatz in [5] and so we take our approximation ansatz for two solitons in the modified NLS system also in the form of the sum of two one soliton fields similar to (3.10). So we take

$$
\psi=\psi_{1}+\psi_{2}=\varphi_{1} e^{i \theta_{1}}+\varphi_{2} e^{i \theta_{2}},
$$

where

$$
\begin{aligned}
& \varphi_{1}=\left(\sqrt{\frac{2+\epsilon}{2}} \frac{a_{1}(t)}{\cosh \left[(1+\epsilon) a_{1}(t)\left(x+\xi_{1}(t)\right)\right]}\right)^{\frac{1}{1+\epsilon}}, \quad \theta_{1}=-\frac{\mu_{1}(t)}{2}\left(x+\frac{\xi_{1}(t)}{2}\right)+a_{1}^{2}(t) t+\lambda_{1}(t), \\
& \varphi_{2}=\left(\sqrt{\frac{2+\epsilon}{2}} \frac{a_{2}(t)}{\cosh \left[(1+\epsilon) a_{2}(t)\left(x+\xi_{2}(t)\right)\right]}\right)^{\frac{1}{1+\epsilon}}, \quad \theta_{2}=-\frac{\mu_{2}(t)}{2}\left(x+\frac{\xi_{2}(t)}{2}\right)+a_{2}^{2}(t) t+\lambda_{2}(t),
\end{aligned}
$$

and $a_{i}(t), \xi_{i}(t), \mu_{i}(t)$ and $\lambda_{i}(t)$ for $i=1,2$ are our collective coordinates. Note that, as is clear from $(3.9), \xi_{i}(t)=(-1)^{i}\left(v t+x_{0}\right)$ when the solitons are far away from each other, so velocity is given by $\mu_{i}(t)$ and is also contained in $\xi_{i}(t)$. This approximation ansatz models two lumps which, when they are far apart, resemble two one-soliton solutions akin to (3.9) with heights $a_{i}(t)$, positions $\xi_{i}(t)$, velocities $\mu_{i}(t)$ and phases $\lambda_{i}(t)$.

In the case $\epsilon=0$ the system is integrable and this ansatz is similar to the one we used in [5] with the additional features of a time dependence in the width of the solitons; also the height, position, velocity and phase of each soliton are allowed to vary independently (whereas previously we insisted that $a_{1}(t)=a_{2}(t), \xi_{1}(t)=-\xi_{2}(t), \mu_{1}(t)=-\mu_{2}(t)$ and $\left.\lambda_{1}(t)=\lambda_{2}(t)\right)$. In particular this allows a previously static parameter, the phase difference between the solitons $\delta \equiv \lambda_{1}-\lambda_{2}$, to vary in time. These changes have been made based on our observations in [5] and we have later found that this improved approximation ansatz gives more accurate results for the NLS solitons when we compare them with our results in [5].

For $\epsilon \neq 0$ and $\delta=n \pi$, where $n \in \mathbb{Z}$, the approximation ansatz (3.15) transforms under the parity defined in (1.1) as in (3.12); thus the field configuration possesses the additional symmetries mentioned before and necessary for the system to be quasi-integrable and so has asymptotically conserved charges.

For $\epsilon \neq 0$ and $\delta \neq n \pi$ the approximation ansatz does not transform under the parity defined in (1.1) as required for quasi-integrability and so the system appears to be nonintegrable and there are no constraints on the charges.

\subsection{Implementing the approximation in modified NLS}

In order to proceed with the collective coordinate approximation we insert our approximation ansatz (3.15) into the Lagrangian (3.1) to obtain an effective Lagrangian:

$$
\mathcal{L}=I_{a_{1}} \dot{a_{1}}+I_{a_{2}} \dot{a_{2}}+I_{\xi_{1}} \dot{\xi_{1}}+I_{\xi_{2}} \dot{\xi_{2}}+I_{\mu_{1}} \dot{\mu_{1}}+I_{\mu_{2}} \dot{\mu_{2}}+I_{\lambda_{1}} \dot{\lambda_{1}}+I_{\lambda_{2}} \dot{\lambda_{2}}-V
$$


where the dot denotes differentiation with respect to time; and the $I$ 's and $V$ are functions of $a_{1,2}(t), \xi_{1,2}(t), \mu_{1,2}(t), \lambda_{1,2}(t)$ and $t$. These functions are fully described in appendix B.

From this effective Lagrangian we derive equations of motion as a set of coupled ODEs of the form, (for different choices of $q$ ):

$\dot{I_{q}}-\dot{a_{1}} \frac{\partial I_{a_{1}}}{\partial q}-\dot{a_{2}} \frac{\partial I_{a_{2}}}{\partial q}-\dot{\xi_{1}} \frac{\partial I_{\xi_{1}}}{\partial q}-\dot{\xi_{2}} \frac{\partial I_{\xi_{2}}}{\partial q}-\dot{\mu_{1}} \frac{\partial I_{\mu_{1}}}{\partial q}-\dot{\mu_{2}} \frac{\partial I_{\mu_{2}}}{\partial q}-\dot{\lambda_{1}} \frac{\partial I_{\lambda_{1}}}{\partial q}-\dot{\lambda_{2}} \frac{\partial I_{\lambda_{2}}}{\partial q}+\frac{\partial V}{\partial q}=0$,

where $q$ denotes the collective coordinates $q=a_{1}, a_{2}, \xi_{1}, \xi_{2}, \mu_{1}, \mu_{2}, \lambda_{1}, \lambda_{2}$. We decouple these equations and solve them using the 4 th order Runge-Kutta method.

\subsection{Results for NLS}

Here we describe the results of our analysis of the scattering of two solitons in our collective coordinate approximation for a range of initial values of the collective coordinates, and compare the results against those given by a full numerical simulation. This allows us to determine the effective range of parameters for our choice of the approximation ansatz. First, we consider the cases when $\epsilon=0$ which correspond to the non-perturbed, integrable NLS. In all our studies we use $\eta=-1$ and take our initial height/width parameter to be $a_{1}=a_{2}=1$. We start our solitons from initial positions $\xi_{1}=-5, \xi_{2}=5$ (i.e. far enough apart not to affect one another initially) and send each one of them towards the other with some initial velocity $v=\mu_{1}=-\mu_{2}$.

As shown in our previous work [5] the solitons' scattering is highly dependent on the relative phase between them, i.e. $\delta \equiv \lambda_{1}-\lambda_{2}$; so initially we compare the solitons' dynamics between the collective coordinate approximation and full numerical simulation for a range of $\delta$. In each case only the trajectory of the right hand soliton has been plotted, calculated by each method, in order to keep the plots clearer. Figure 1 compares the trajectories for solitons with initial velocity $v=0.01$ and initial phase difference $\delta=0, \frac{\pi}{4}, \frac{\pi}{2}, \frac{3 \pi}{4}, \pi$ (the results are symmetric around $\pi$ and periodic in $2 \pi$ ). This figure shows that for most values of $\delta$ (whenever $\delta \neq 0$ ) both approaches produce almost identical trajectories, with a percentage difference in their trajectories at $t=250$ (i.e. a significant time after the solitons' collision) of $0.53 \%$ for $\delta=\frac{\pi}{4} ; 0.026 \%$ for $\delta=\frac{\pi}{2} ; 0.0067 \%$ for $\delta=\frac{3 \pi}{4}$; and $0.0039 \%$ for $\delta=\pi$. It is evident from these values that the earlier the solitons repel the more accurate the approximation is.

In the case of $\delta=0$ both results show excellent qualitative agreement for the first 3 oscillations, but the solitons in the collective coordinate approximation break away from oscillating around each other much earlier than in the full simulation. However, the qualitative results remain the same, and one possible cause for any disagreement between the collective coordinate approximation and full simulation is because in the full simulation the solitons deform one another away from the form given by (3.15) when they are in close proximity and the collective coordinate approximation does not allow such a deformation.

When we compare the trajectories of the solitons as calculated using each method we can directly compare our collective coordinates $\xi_{i}(t), i=1,2$ with the positions of the solitons in the full numerical simulation (i.e. the position of the maxima of the soliton wavefunction). It is not generally possible to make a direct comparison between other 


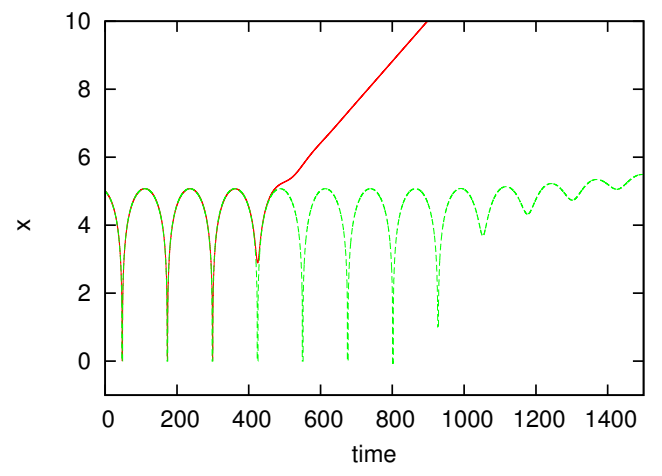

(a)

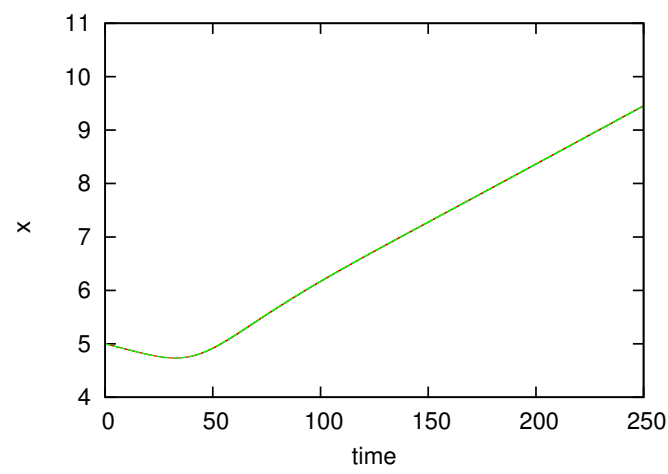

(c)

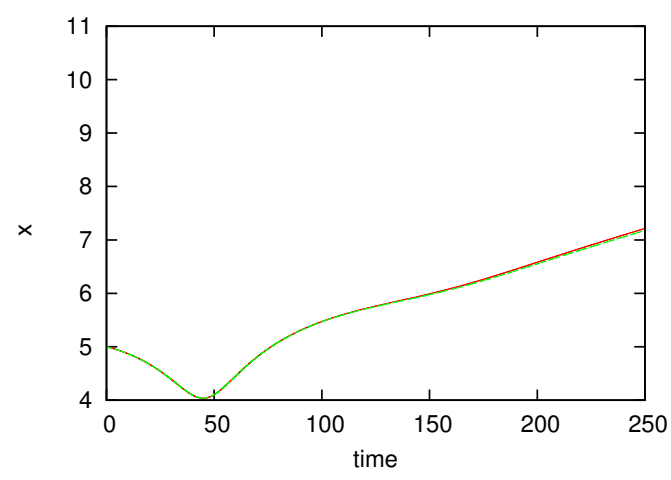

(b)

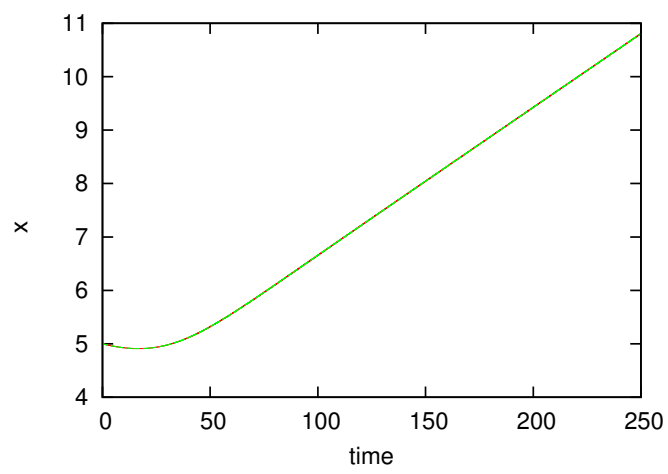

(d)

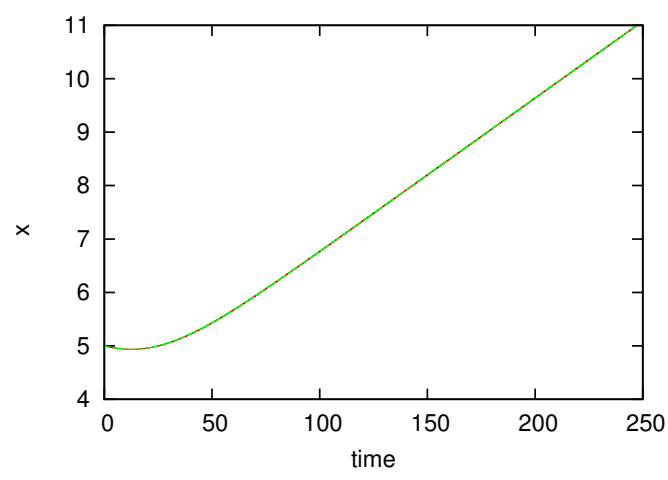

(e)

Figure 1. The distance of a soliton from the centre of mass of a system as a function of time. The system consists of two solitons initially placed at \pm 5 and sent towards their centre of mass with an initial velocity $v=0.01$. Initial height/width parameter of each soliton is 1 and the initial phase difference between them is: (a) $\delta=0$, (b) $\delta=\frac{\pi}{4}$, (c) $\delta=\frac{\pi}{2}$, (d) $\delta=\frac{3 \pi}{4}$, (e) $\delta=\pi$. For each plot the solid line has been obtained using the collective coordinate approximation and the dashed line is the result of the full simulation (these may be indistinguishable). 
collective coordinates and physical measurables of the solitons in the full simulation. For example, if we consider our ansatz (3.15) we can see that $|\psi|^{2}=\varphi_{1}^{2}+\varphi_{2}^{2}+2 \varphi_{1} \varphi_{2} \cos \left(\theta_{1}-\theta_{2}\right)$; for the solitons far apart $2 \varphi_{1} \varphi_{2} \cos \left(\theta_{1}-\theta_{2}\right)$ will be negligible and the heights of each soliton will correspond to $\sqrt{\frac{2+\epsilon}{2}} a_{i}^{\frac{1}{1+\epsilon}}, i=1,2$ but when the solitons come closer together $2 \varphi_{1} \varphi_{2} \cos \left(\theta_{1}-\theta_{2}\right)$ is no longer negligible and the height of each soliton has some contribution from the interacting part. Similarly the coordinates $\lambda_{i}(t), i=1,2$ give the initial phase difference as $\lambda_{1}-\lambda_{2}$ but as the solitons come together there are contributions to the phase difference from the other coordinates ( $\operatorname{consider} \theta_{1}-\theta_{2}$ ). Therefore, to check the validity of the collective coordinate approximation using our other coordinates we must consider them in combination and compare the heights and phase difference of the solitons.

So, we further explore the usefulness of the collective coordinate approximation for $\epsilon=0$ by comparing the heights of the solitons as they collide, calculated using the collective coordinate approximation and using the full numerical simulation, for a variety of initial values of phase difference $\delta$. Figure 2 compares the heights of the solitons during collision, calculated by each method, for solitons with initial velocity $v=0.01$ and initial phase difference $\delta=\frac{\pi}{4}, \frac{\pi}{2}$. It is clear that in the cases where the trajectories show excellent qualitative agreement, i.e. when $\delta=\frac{\pi}{4}, \frac{\pi}{2}$, the heights of the solitons also show excellent qualitative agreement. This can be seen from figure 2 where the plotted lines are essential coincident, and from the percentage difference in the heights of the solitons as calculated by each method which, at $t=250$, is of the order $10^{-3 \%}$ (comparing the right and left hand solitons separately). Figure 3 is similar to figure 2 but with initial phase difference $\delta=0$, and only the heights of the right hand solitons have been plotted to maintain clarity (the results for the heights of the left hand solitons are similar). In this case the heights of the solitons peak when the solitons come together in a similar way in both the approximation and the full simulation; the heights of the solitons in the approximation cease peaking when the solitons cease to oscillate around each other which happens earlier in the collective coordinate approximation than in the full simulation (for the trajectories see figure 1(a)). These observations support those drawn when comparing the trajectories of the solitons.

We also consider the phase difference between the solitons as they collide to gain more information. Figure 4 compares the phase difference between the solitons during collision, as calculated by each method, for solitons with initial velocity $v=0.01$ and initial phase differences $\delta=0, \frac{\pi}{2}$. This figure shows excellent agreement in the case $\delta=\frac{\pi}{2}$, the percentage difference in the results at $t=250$ is $0.87 \%$. When $\delta=0$ the phase difference between the solitons in the collective coordinate approximation varies around zero between $\pm \frac{\pi}{4}$ as the solitons come together then increases as the solitons repel (for the trajectory see figure 1(a)), in the full numerical simulation the phase difference varies when the solitons come together but only by $\pm 5 \times 10^{-5}$. The dissimilarity between the phase difference in the two methods for solitons starting in the most attractive channel $(\delta=0)$ suggests that the approximation struggles to model exactly how the phase difference changes as the solitons come close together, this may explain the small differences in the physical properties of the solitons that have been noted in the $\delta=0$ case. 


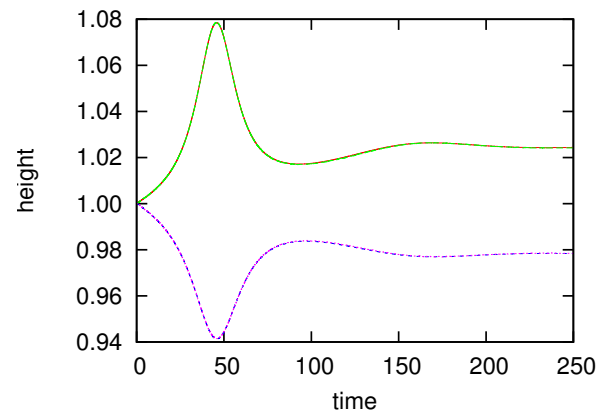

(a)

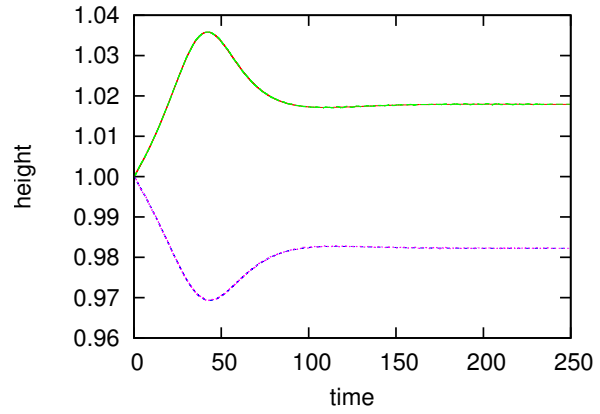

(b)

Figure 2. The heights of colliding solitons as a function of time. The system consists of two solitons initially placed at \pm 5 and sent towards their centre of mass with an initial velocity $v=0.01$. Initial height/width parameter of each soliton is 1 and the initial phase difference between them is: (a) $\delta=\frac{\pi}{4}$, (b) $\delta=\frac{\pi}{2}$. For each plot the increasing lines are the results for the right hand solitons for the collective coordinate approximation (solid line) and the full simulation (long dashed line), these are very similar. The decreasing lines are results for the left hand soliton for the collective coordinate approximation (short dashed line) and the full simulation (dotted line), these are also very similar.

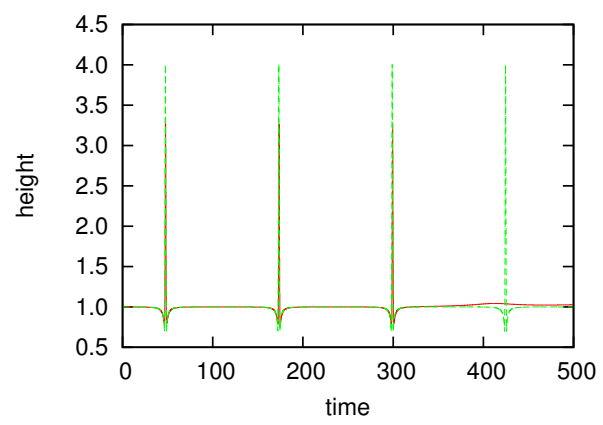

Figure 3. The heights of colliding solitons as a function of time. The system consists of two solitons initially placed at \pm 5 and sent towards their centre of mass with an initial velocity $v=0.01$. Initial height/width parameter of each soliton is 1 and the initial phase difference between them is $\delta=0$. The solid line has been obtained using the collective coordinate approximation and the dashed line is the result of the full simulation (for $t$ up to 350 these are difficult to distinguish).

Next we consider the effect of the initial velocity on the accuracy of the collective coordinate approximation. Figure 5 compares the trajectories given by the collective coordinate approximation and those given by the full numerical simulation for solitons with initial phase difference $\delta=\frac{\pi}{4}$ and initial velocity $v=0.1$ and $v=0.2$. Although we expect the collective coordinate approximation to be better at smaller velocities, as mentioned in section 2.1, the total effect of the initial velocity on the accuracy of the collective coordinate approximation is difficult to gauge in full generality. This is because, when the initial velocity is changed, the amount of time the solitons spend close together during their interaction 


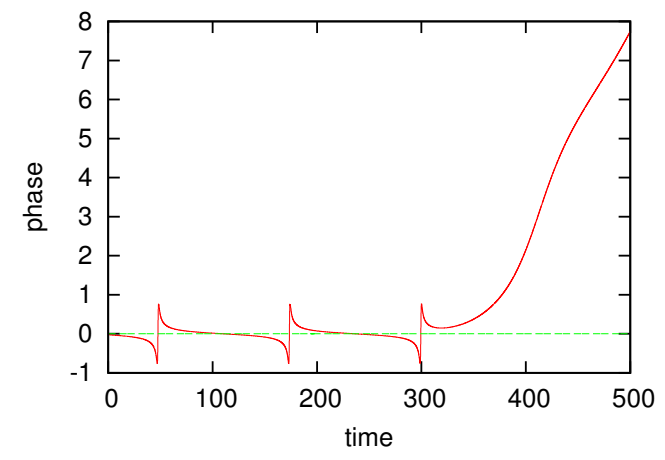

(a)

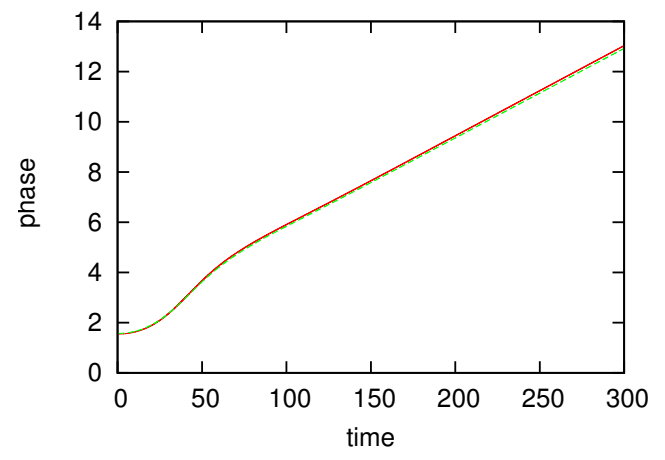

(b)

Figure 4. The phase difference between solitons as a function of time. The system consists of two solitons initially placed at \pm 5 and sent towards their centre of mass with an initial velocity $v=0.01$. Initial height/width parameter of each soliton is 1 and the initial phase difference between them is: (a) $\delta=0$ and (b) $\delta=\frac{\pi}{2}$. For each plot the solid line has been obtained using the collective coordinate approximation and the dashed line is the result of the full simulation (these may be indistinguishable).

changes which, as we have already surmised, affects the accuracy of the approximation. Figure 5 shows that, as expected, increasing initial velocity decreases the accuracy of the approximation slightly, with a percentage difference in position of the solitons a significant time after their collision of $1.3 \%$ for initial velocity $v=0.1$ (at $t=100$ ) and $3.9 \%$ for initial velocity $v=0.2$ (at $t=50$ ), though for solitons which do not spend much time close together the approximation is still very good up to an initial velocity of at least $v=0.2$. As the collective coordinate approximation assumes slow moving solitons (see section 2.1) our results show that the approximation is extremely reliable for low velocity and for higher velocities it is more reliable than could have been reasonably expected. As the collective coordinate approximation neglects any radiative corrections to the solitons, this agreement for velocities of up to $v=0.2$ suggests that the radiative corrections are small for these initial values.

\subsection{Results for modified NLS}

In the case $\epsilon \neq 0$ the modified NLS system of two solitons is no longer integrable; this means that the system no longer has an infinite number of conserved quantities and so some energy can be lost as radiation during soliton interactions. As before each simulation starts with solitons with initial positions $\xi_{1}=-5, \xi_{2}=5$, initial height/width parameter $a_{1}=a_{2}=1$, and various initial phase differences and velocities towards each other. As in the $\epsilon=0$ case we find that the accuracy of the approximation depends on the amount of time the solitons spend in close proximity of each other during their interaction. This can be seen in figure 6 which compares the trajectories of solitons with initial velocities $v=0.01, \epsilon= \pm 0.06$ and $\delta=0, \frac{\pi}{4}, \frac{\pi}{2}$ (plots for $\delta=\frac{3 \pi}{4}, \pi$ show excellent agreement so are not included). We note that for $\epsilon= \pm 0.06$ and $\delta \neq 0$ the results of the collective coordinate 


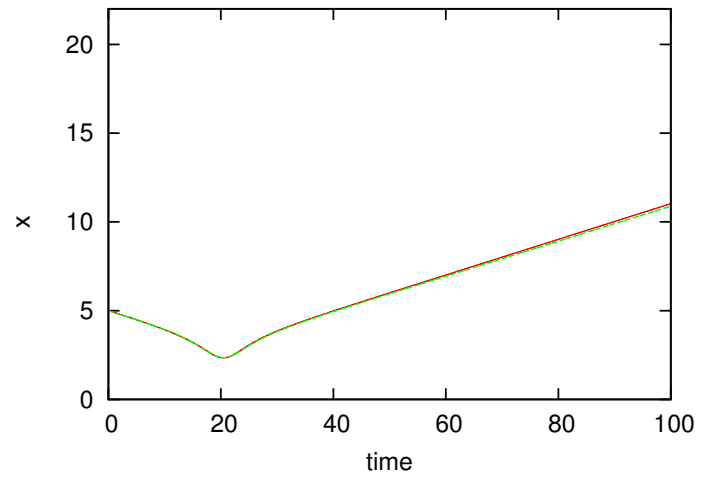

(a)

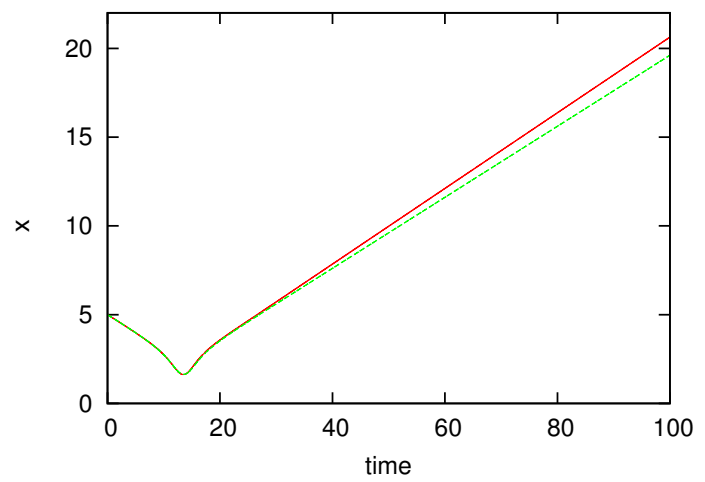

(b)

Figure 5. The distance of a soliton from the centre of mass of a system as a function of time. The system consists of two solitons initially placed at \pm 5 , with initial height/width parameter of 1 and the initial phase difference between them of $\delta=\frac{\pi}{4}$. The solitons move towards the centre of mass with initial velocity (a) $v=0.1$, and (b) $v=0.2$. For each plot the solid line describes the outcome obtained in the collective coordinate approximation and the dashed line shows the result of the full simulation (these may be indistinguishable).

approximation show excellent qualitative agreement with the results of the full numerical simulation. The percentage difference in the positions of the solitons at a significant time after the collision for initial phase difference $\delta=\frac{\pi}{4}$ is $9.4 \%$ for $\epsilon=0.06$ (at $t=400$ ) and $0.29 \%$ for $\epsilon=-0.06$ (at $t=250$ ); the percentage difference in the positions of the solitons for initial phase difference $\delta=\frac{\pi}{2}$ is $0.034 \%$ for $\epsilon=0.06$ (at $t=250$ ) and $0.041 \%$ for $\epsilon=-0.06$ (at $t=250$ ). However, for $\epsilon= \pm 0.06$ and $\delta=0$ the differences between the approximation and full simulation are more pronounced than in the $\epsilon=0$ case: the collective coordinate approximation accurately describes the initial coming together of the solitons, but it does not capture the decreasing amplitude and increasing frequency of the oscillations demonstrated by the full simulation before the solitons eventually repel. This increased difference is probably because, for the $\epsilon \neq 0$ case, the solitons deform each other to a greater extent as they approach each other and some energy is radiated out which is not accounted for in the approximation.

The amount of energy lost by the solitons in the full simulation is shown in figure 7 where we plot the energy of the system during a scattering for $\epsilon=0.06$, and $\delta=0, \frac{\pi}{4}, \frac{\pi}{2}$ and for the same initial conditions as those used in the trajectory plots (plots for $\epsilon=-0.06$ are very similar), and we do not plot the results of the collective coordinate approximation as this approximation does not allow the loss of energy. Over time the cases $\delta=\frac{\pi}{4}, \frac{\pi}{2}$ demonstrate an incredibly small energy change; when $t=300$ (i.e. at a significant time after any collisions) they both have a percentage energy loss of $1.4 \times 10^{-6 \%}$. In the case $\delta=0$ the energy is constant until the solitons come together at which point some energy is radiated out, the system then evolves as two separate solitons and emits some small energy waves which we absorb as they reach the boundary so we see that the total energy of the soliton decreases. The percentage energy loss in this case is $1.1 \%$. 


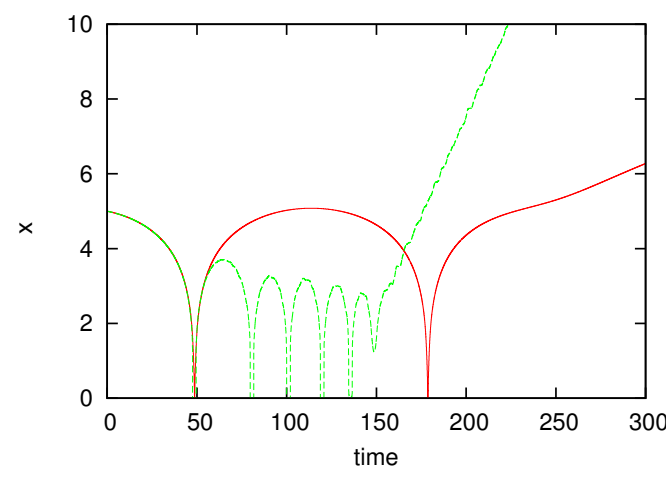

(a)

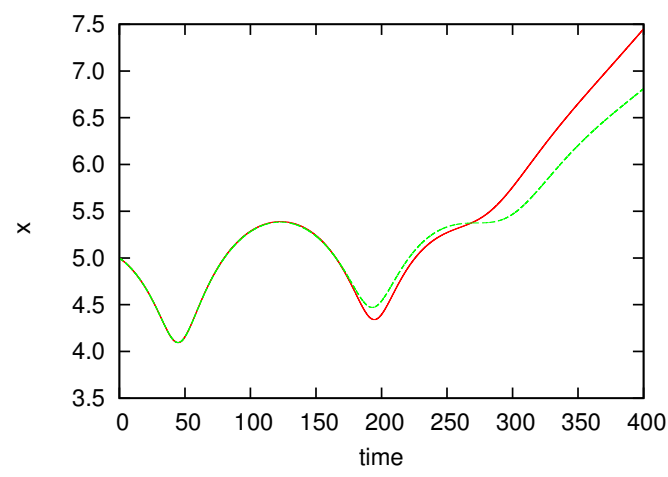

(c)

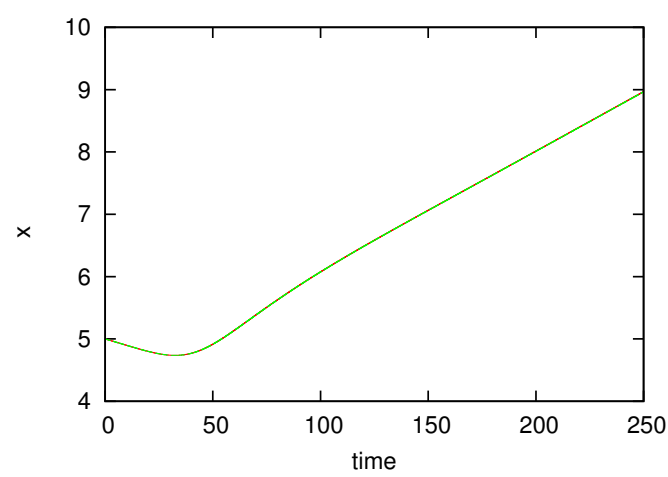

(e)

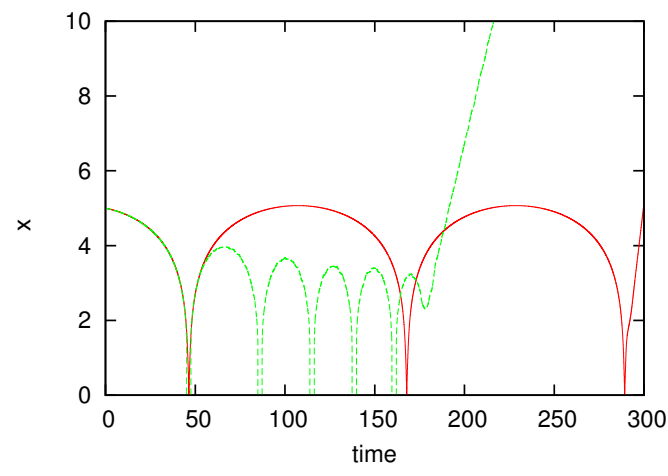

(b)

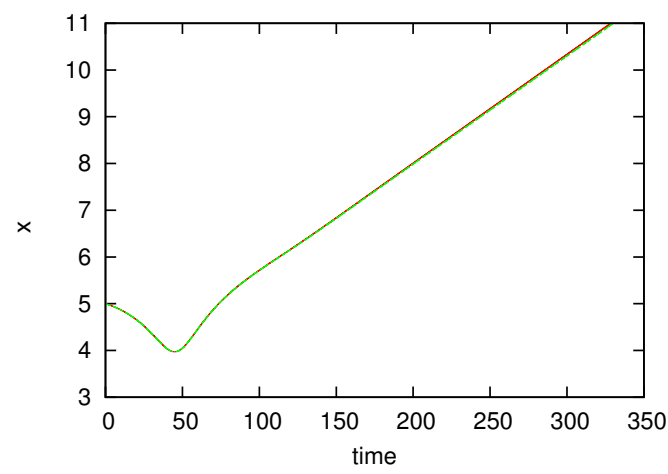

(d)

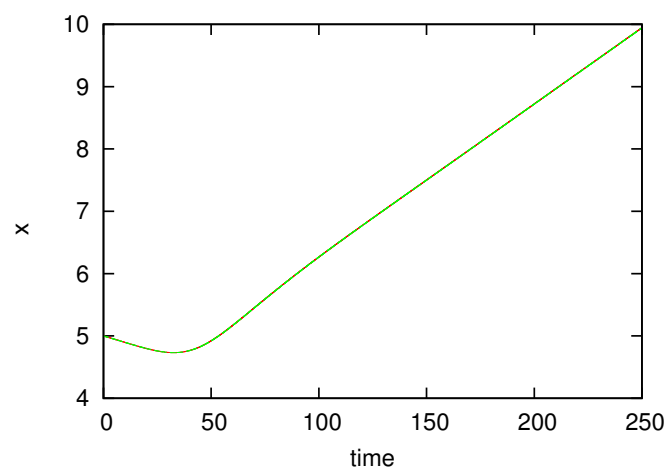

(f)

Figure 6. The distance of a soliton from the centre of mass of a system as a function of time. The system consists of two solitons initially placed at \pm 5 each with an initial velocity of $v=0.01$ towards the centre of mass. Initial height/width parameter of each soliton is 1 with $\delta=0$ and (a) $\epsilon=0.06$, (b) $\epsilon=-0.06 ; \delta=\frac{\pi}{4}$ and (c) $\epsilon=0.06$, (d) $\epsilon=-0.06 ; \delta=\frac{\pi}{2}$ and (e) $\epsilon=0.06$, (f) $\epsilon=-0.06$. For each plot the solid line is result of the collective coordinate approximation and the dashed line is the result of the full simulation (these may be indistinguishable). 


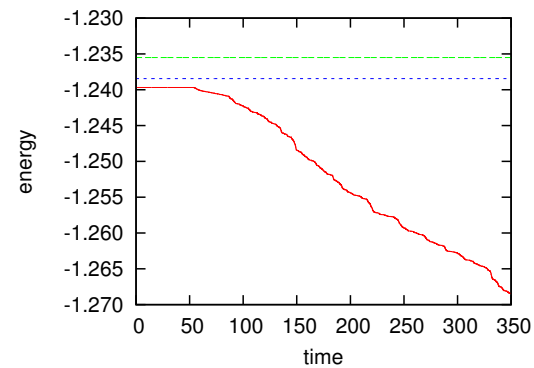

Figure 7. The time dependency of the energy of the solitons for $\epsilon=0.06$ placed initially at \pm 5 . Each soliton is of initial height/width parameter of 1 and is sent towards the centre of mass with initial velocity $v=0.01 . \delta=0$ corresponds to the solid line, $\delta=\frac{\pi}{4}$ the dotted line and $\delta=\frac{\pi}{2}$ the dashed line.

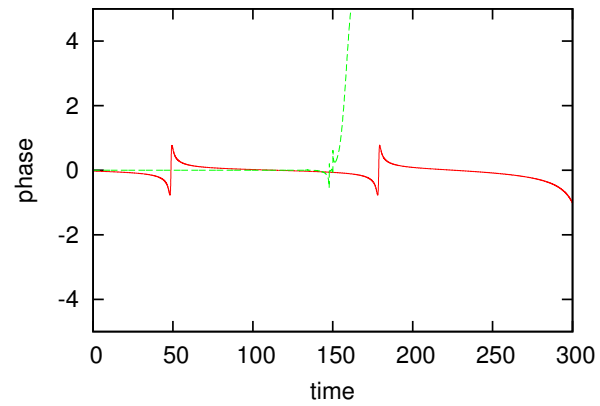

Figure 8. The phase difference between solitons as a function of time. The system consists of two solitons initially placed at \pm 5 and sent towards their centre of mass with an initial velocity $v=0.01$. Initial height/width parameter of each soliton is 1 and the initial phase difference between them is $\delta=0$. The solid line has been obtained using the collective coordinate approximation and the dashed line is the result of the full simulation.

As in the $\epsilon=0$ case we consider the phase difference between the solitons as they collide. Figure 8 compares the phase difference between the solitons during collision, as calculated by each method, for solitons with $\epsilon=0.06$, initial velocity $v=0.01$ and initial phase difference $\delta=0$. This figure resembles the one for the $\epsilon=0$ case: the phase difference calculated using the approximation peaks when the solitons come together and steadily increases when the solitons cease oscillating around each other, the phase difference calculated using the full simulation has very small peaks when the solitons come together and also steadily increases when the solitons repel. Comparing this figure to figure 4 shows that the change in $\epsilon$ has no obvious affect on the how well the collective coordinate approximation models the time dependence of the phase difference.

We also consider the effect of the initial velocity on the accuracy of the collective coordinate approximation when $\epsilon=0.06$. Figure 9 compares the trajectories obtained in the collective coordinate approximation and those found by the full numerical simulation for solitons with initial values as in figure 5 but with $\epsilon=0.06$. These results show that the 


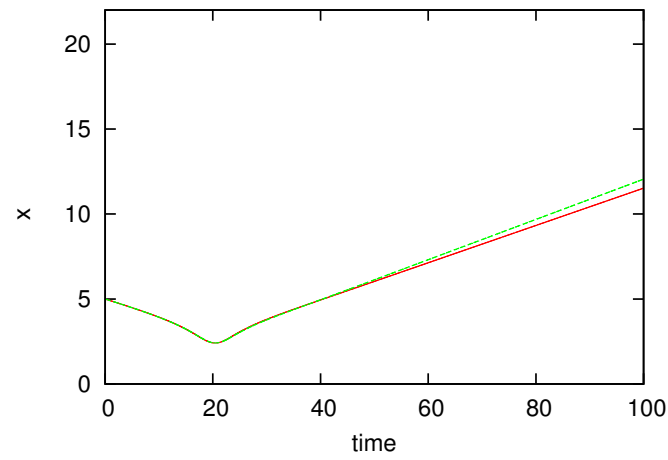

(a)

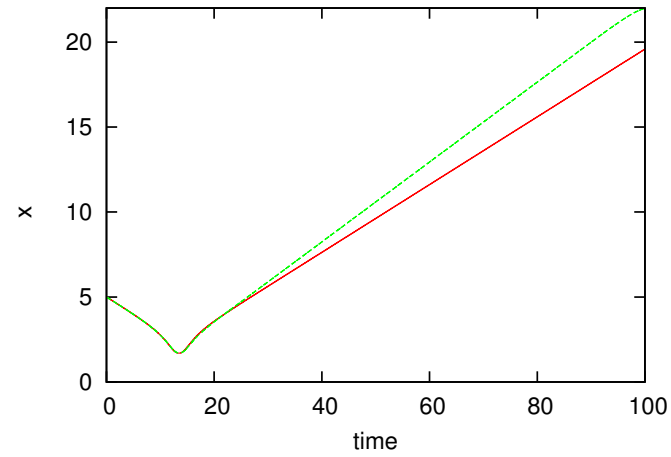

(b)

Figure 9. The distance of a soliton from the centre of mass of a system as a function of time with $\epsilon=0.06$. The system consists of two solitons initially placed at \pm 5 , with initial height/width parameter of 1 and the initial phase difference between them of $\delta=\frac{\pi}{4}$. The solitons move towards the centre of mass with initial velocity (a) $v=0.1$, and (b) $v=0.2$. For each plot the solid line is result of the collective coordinate approximation and the dashed line is the result of the full simulation.

accuracy of the approximation is still quite good up to $v=0.2$, with percentage difference in position of the solitons of $4.4 \%$ for $v=0.1$ (at $t=100$ ) and $9.2 \%$ for $v=0.2$ (at $t=50$ ). The trajectories of the solitons for these initial values show slightly less agreement than in the equivalent simulations with $\epsilon=0$ (this can be seen by comparing figures 9 and 5 ).

Next we increase the parameter $\epsilon$ to investigate its effect on the accuracy of the approximation. Figure 10 presents the plots of the trajectories derived in the collective coordinate approximation and the full numerical simulation for solitons with initial phase difference $\delta=\frac{\pi}{4}$, and various values of $\epsilon$ and initial velocity. This figure also shows that, for solitons which do not spend much time in close proximity of each other, increasing the value of $\epsilon$ reduces the accuracy of the approximation very slightly with excellent qualitative agreement up to at least $\epsilon=0.3$, the percentage difference in the positions of the solitons is $0.51 \%$ for $\epsilon=0.1, v=0.01$ at $t=250$, and $1.4 \%$ for $\epsilon=0.3, v=0.1$ at $t=100$.

So we have learnt that the collective approximation reproduces the results of the full simulation well when the solitons do not come too close to each other. But how good is this 'well' - can we quantify it? To do this we performed many simulations for the solitons sent towards each other in configurations corresponding to their initial phases not being in an attractive channel and we carried this out for a range of small values of $\varepsilon$.

We quickly found that there was only a smallish range of $\varepsilon$, namely $|\varepsilon|<0.9$ over which the soliton system roughly resembled the behaviour of the integrable $\varepsilon=0$ case (with small modifications as described above). Of course, this does not mean that the system does not have soliton like solutions for larger values of $|\varepsilon|$. We only have found that the numerical schemes we used to study their behaviour ran into problems. Of course, our schemes were quite involved, as we described in section 2.2 , the conclusion we can draw from these results is that either we need even more sophisticated numerical schemes or that the systems were not stable (i.e. even small perturbations could destroy them, and 


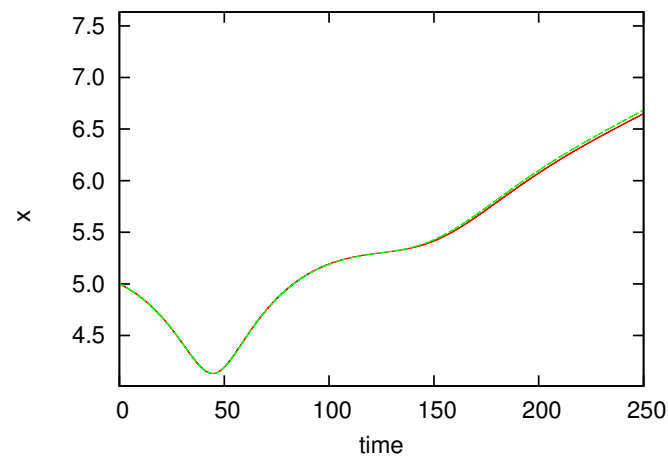

(a)

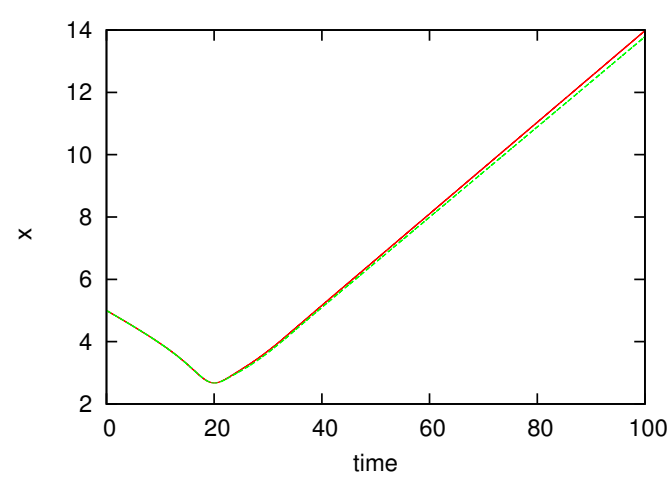

(b)

Figure 10. The distance of a soliton from the centre of mass of a system as a function of time. The system consists of two solitons initially placed at \pm 5 , with initial height/width parameter of 1 and the initial phase difference between them of $\delta=\frac{\pi}{4}$. For (a) the solitons have initial velocity $v=0.01$ and $\epsilon=0.1$, and for (b) the solitons have initial velocity $v=0.1$ and $\epsilon=0.3$. For each plot the solid line is result of the collective coordinate approximation and the dashed line is the result of the full simulation.

in the schemes we used they did destroy them). The later aspect is supported by the fact that the collective approximation did not work for those values of $|\varepsilon|$ either. As we said earlier, this approximation is only an approximation which is less reliable when solitons can deform themselves and when the scattering process emits energy. Hence our feeling is that for larger values of $|\varepsilon|$ the system is not stable, but we have no real 'proof' of this.

So we restricted our attention to smaller values of $|\varepsilon|$. We performed systematically detailed simulations for two values of the initial relative phase between the solitons, namely $\delta=\frac{\pi}{4}$ and $\delta=\frac{\pi}{2}$.

The results demonstrated a good agreement between those of the collective coordinate approximation and of the full simulation. This 'goodness' showed itself in the similar trajectories of the solitons seen in both approaches. This was true when the solitons basically reflected and when they performed a more exotic motion in close proximity of each other before the repulsion. In figure 11 we present 3 plots for $\delta=\frac{\pi}{4}$ for $\varepsilon=0.6, \varepsilon=0.7$ and $\varepsilon=0.8$. The first case demonstrates an almost exact perfect agreement between the results of the full simulation and the collective approximation, the second one is still very good with a slight difference in the variation of velocities of the outgoing solitons and the third one still captures the main features of the process, in particular in the early phase of it, i.e. until approximately $t \sim 150$.

In table 1 we give the values of the relative difference of the trajectories evaluated as the percentage difference in the trajectory calculated using the approximation and using the full simulation a significant time after the solitons interact.

These results, supplemented by the plots, show that the approximation does capture the main features of the scattering and it works amazingly well. However, as the differences are extremely small the results in the tables have to be taken with a pinch of salt. Both data sets are obtained numerically. They both have some unavoidable numerical errors. 


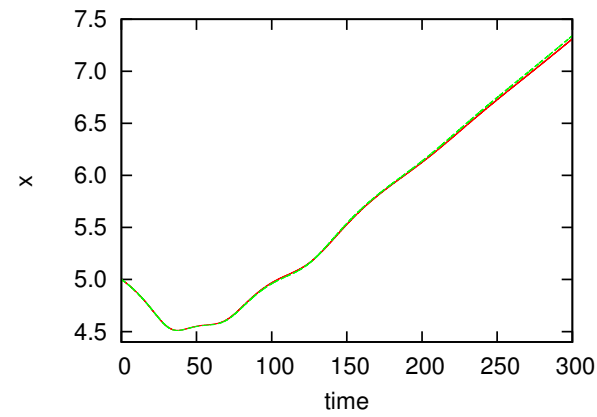

(a)

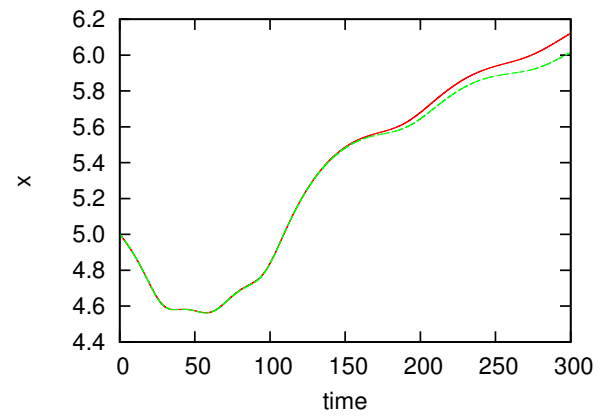

(b)

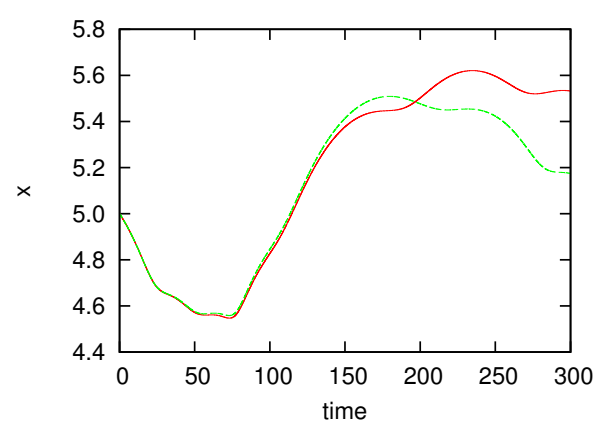

(c)

Figure 11. The distance of a soliton from the centre of mass of a system as a function of time. The system consists of two solitons initially placed at \pm 5 , with initial height/width parameter of 1 and the initial phase difference between them of $\delta=\frac{\pi}{4}$. The value of $\epsilon$ is (a) $\epsilon=0.6$; (b) $\epsilon=0.7$ and (c) $\epsilon=0.8$. For each plot the solid line is result of the collective coordinate approximation and the dashed line is the result of the full simulation.

Of course, we have tried hard to minimise them but some errors are still there. What is important is what we see i.e. that the collective approximation works so well that it manages to capture qualitative features of the scattering when the soliton come close together (but not too close), and when they come very close it still captures some features.

Of course the collective coordinate approximation is still only an approximation in that an infinite number of degrees of freedom had been replaced by only a few ones and our results show that the field theory solitons behave a bit like objects described by very few degrees of freedom and so allow us to think about soliton scatterings in a more physical way. Hence this approximation not only reduces the time need for simulations but also presents a more physical picture of what is going on and as such can lead to a better physical understanding of solitons. A by product of our simulations is the practical (as compared to mathematical) proof of stability of solitons. The numerical simulations always involve small perturbations, resulting from discretisation. As the numerical errors, which we have tried to minimise as much as possible, tend to be random we see that the solitons are not sensitive to such small perturbations, and the agreement with the collective approximation (which 


\begin{tabular}{|r|l|l|}
\hline$\epsilon$ & $\begin{array}{l}\text { Percentage difference } \\
\text { for } \delta=\frac{\pi}{4}\end{array}$ & $\begin{array}{l}\text { Percentage difference } \\
\text { for } \delta=\frac{\pi}{2}\end{array}$ \\
\hline-0.55 & 0.6003 & 0.2090 \\
\hline-0.45 & 0.0928 & 0.0794 \\
\hline-0.10 & 1.0785 & 0.1164 \\
\hline-0.06 & 1.2747 & 0.1314 \\
\hline 0 & 0.5303 & 0.0067 \\
\hline 0.06 & 1.1512 & 0.1812 \\
\hline 0.10 & 2.3001 & 0.1996 \\
\hline 0.45 & 1.0514 & 0.0002 \\
\hline 0.50 & 3.3382 & 0.0312 \\
\hline 0.55 & 1.4303 & 0.0669 \\
\hline 0.60 & 0.3180 & 0.1009 \\
\hline 0.70 & 0.9266 & 0.1091 \\
\hline 0.80 & 3.1927 & 0.5705 \\
\hline
\end{tabular}

Table 1. The percentage difference between the position of a soliton calculated using the collective coordinate approximation and using the full numerical simulation for a range of values of $\epsilon$ and for $\delta=\frac{\pi}{4}$ and $\delta=\frac{\pi}{2}$. For each case the difference is calculated a significant time after the solitons' collision. For $\delta=\frac{\pi}{4}$ and $\epsilon<0$ the difference is calculated at $t=150$, and for $\delta=\frac{\pi}{4}$ and $\epsilon \geqslant 0$ the difference is calculated at $t=250$ as for these values the solitons have a longer interaction. For $\delta=\frac{\pi}{2}$ and all values of $\epsilon$ the difference is calculated at $t=250$.

has additional errors, and different numerical ones) demonstrates the physical robustness of such solitons. Analytical results are very useful but their stability is also extremely important if these structures are ever meant to describe physical systems, which always possess small perturbations. Our simulations have shown that the the solitons of the unmodified and not too strongly modified nonlinear Schrödinger system are stable and their dynamics can be described in part by the collective coordinate approximation model.

In our numerical simulations we calculate and compare the quasi-conservation of the first non-trivial charge beyond the energy and momentum, i.e. the charge $Q^{(4)}$ defined in (3.7). We do this by computing the corresponding anomaly $\beta_{4}$, defined in (3.7) ( $X$ is defined in (3.5) and an explicit expression for $\alpha^{(3,-4)}$ is given in appendix (A)), and by integrating it over time to get the integrated anomaly:

$$
\begin{aligned}
\chi^{(4)}(t) & \equiv \int_{-\infty}^{t} d t^{\prime} \beta_{4}=\int_{-\infty}^{t} d t^{\prime} \int_{-\infty}^{\infty} d x X \alpha^{(3,-4)} \\
& =-2 i \int_{-\infty}^{t} d t^{\prime} \int_{-\infty}^{\infty} d x\left((\epsilon+1) R^{\epsilon}-1\right) \partial_{x} R\left[-6 R^{2}+\frac{3}{2}\left(\partial_{x} \varphi\right)^{2} R-2 \partial_{x}^{2} R+\frac{3}{2} \frac{\left(\partial_{x} R\right)^{2}}{R}\right] .
\end{aligned}
$$

This is then computed in terms of the fields $R$ and $\varphi$ which are defined by writing each soliton field $\psi$ in the form $\psi \equiv \sqrt{R} e^{i \frac{\varphi}{2}}$. 
In figure 12 we present the plots of the time-integrated anomaly for each of the trajectories shown in figure 6 . We note that the results are very similar although not as exact as some of the trajectories. The time integrated anomalies are most different in the case $\delta=0$ as the collective coordinate approximation shows a distinct peak when the solitons come together when compared to the results seen in the full simulation which displays only a minute deviation from zero at these points (of the order $10^{-7}$ ). However, when the solitons are far apart the time-integrated anomaly does return to zero as predicted in [2] when $\delta$ is an integer value of $\pi$, as this corresponds to the case when the parity symmetry described in (3.12) is present. When $\delta$ is not an integer multiple of $\pi$ this symmetry is not present and the integrated anomalies do not return to zero, and the collective coordinate method shows similar time-integrated anomalies to those found in the full simulation. This shows that, in addition to the trajectories, the collective coordinate approximation also does reproduce quite well the results for the anomalies obtained using the full numerical method.

\section{The modified sine-Gordon model}

So far we have looked at the scattering of solitons in modified NLS models, i.e. models in which the solitons are nontopological and we have demonstrated the usefulness of using our collective coordinate approximation as a tool to investigate their properties. But one may ask if this is also the case for models which involve topological solitons; this is what we consider in this section. We base our discussion on the example of a modified sineGordon model.

So we consider the Lagrangian given by

$$
L=\int d x \frac{1}{2}\left(\left(\partial_{t} \psi\right)^{2}-\left(\partial_{x} \psi\right)^{2}\right)-V(\psi)
$$

For the sine-Gordon potential $V=V_{\mathrm{SG}}=\frac{1}{8} \sin ^{2}(2 \psi)$ there are static one-soliton solutions of the form

$$
\psi=\operatorname{ArcTan}\left(e^{ \pm\left(x-x_{0}\right)}\right) .
$$

A modification on this model was suggested in [3] by taking a change of variable $\psi \rightarrow \phi$ given by

$$
\psi(\phi)=\frac{c \phi}{\sqrt{1+\epsilon \phi(\phi-2 \gamma)}},
$$

which has two free parameters $\epsilon$ and $\gamma$, and the parameter $c$ chosen to be

$$
c=\sqrt{1+\epsilon \pi\left(\frac{\pi}{4}-\gamma\right)},
$$

such that $\phi(\psi=0)=0$ and $\phi\left(\psi=\frac{\pi}{2}\right)=\frac{\pi}{2}$.

Then $\phi$, obtained by calculating $\phi=\phi(\psi)$ from (4.3) and using $\psi$ given by (4.2), is a solution of the static Euler-Lagrangian equation associated to (4.1) with the potential

$$
V(\phi)=\left(\frac{d \phi}{d \psi}\right)^{2} V_{\mathrm{SG}}=\frac{1}{8} \frac{(1+\epsilon \phi(\phi-2 \gamma))^{3}}{c^{2}(1-\epsilon \gamma \phi)^{2}} \sin ^{2}(2 \psi(\phi)) .
$$




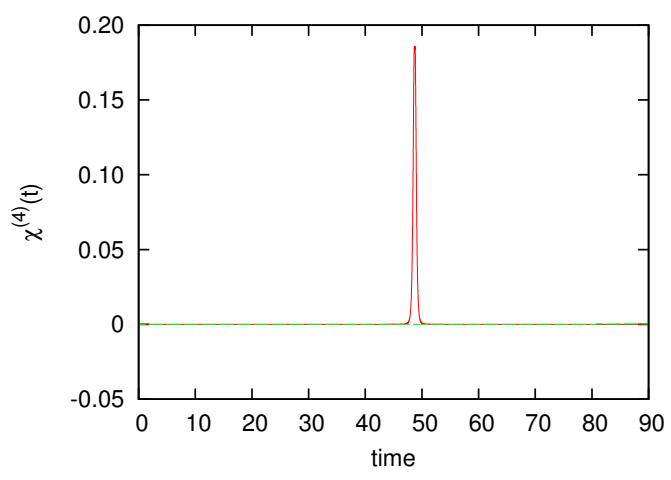

(a)

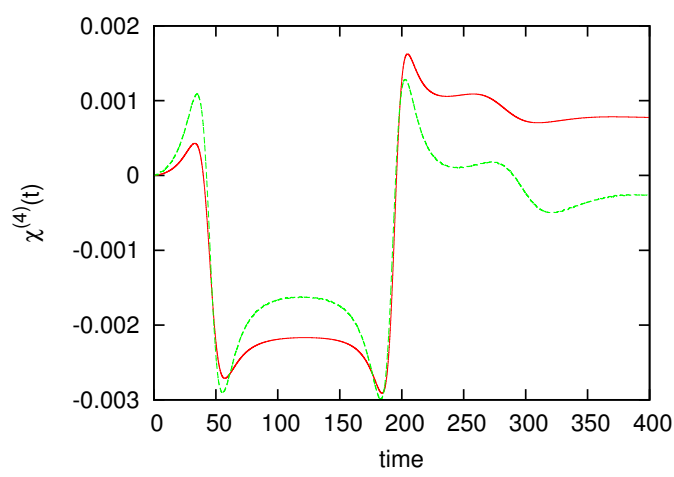

(c)

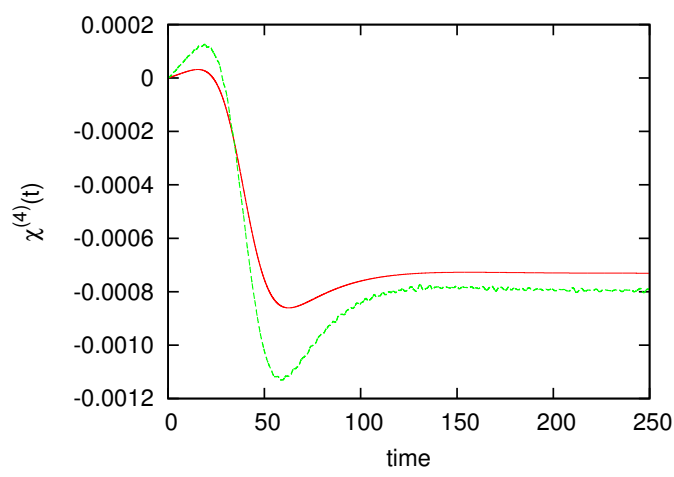

(e)

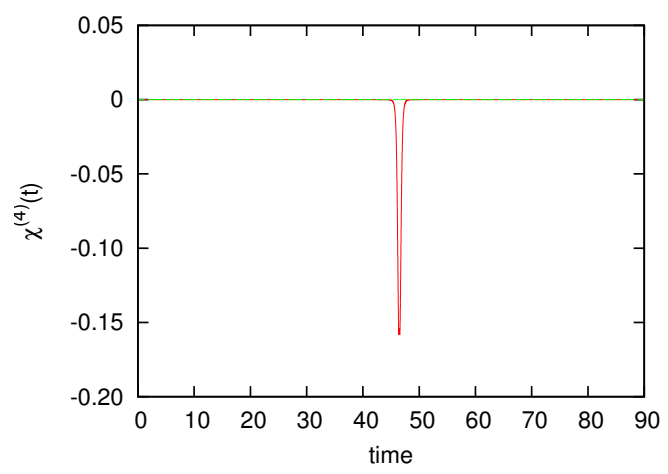

(b)

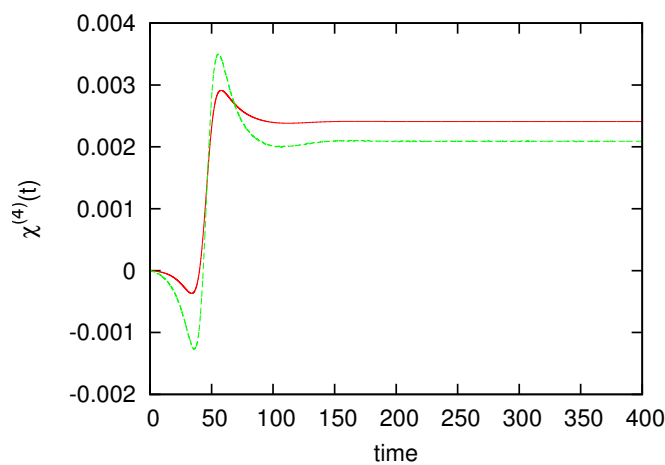

(d)

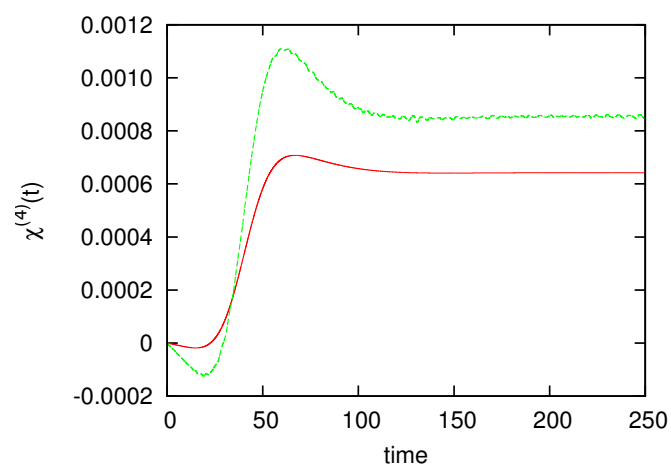

(f)

Figure 12. The time-integrated anomaly, $\chi^{(4)}(t)$, for the soliton interactions shown in figure 6 with $\delta=0$ and (a) $\epsilon=0.06$, (b) $\epsilon=-0.06 ; \delta=\frac{\pi}{4}$ and (c) $\epsilon=0.06$, (d) $\epsilon=-0.06 ; \delta=\frac{\pi}{2}$ and (e) $\epsilon=0.06$, (f) $\epsilon=-0.06$. For each plot the solid line is result of the collective coordinate approximation and the dashed line is the result of the full simulation. 


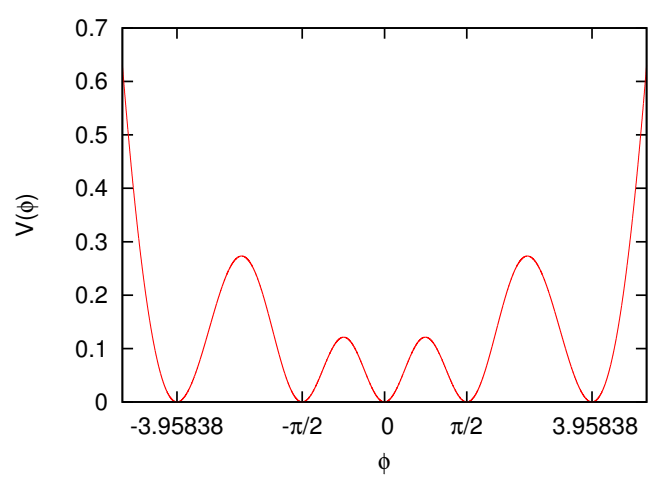

(a)

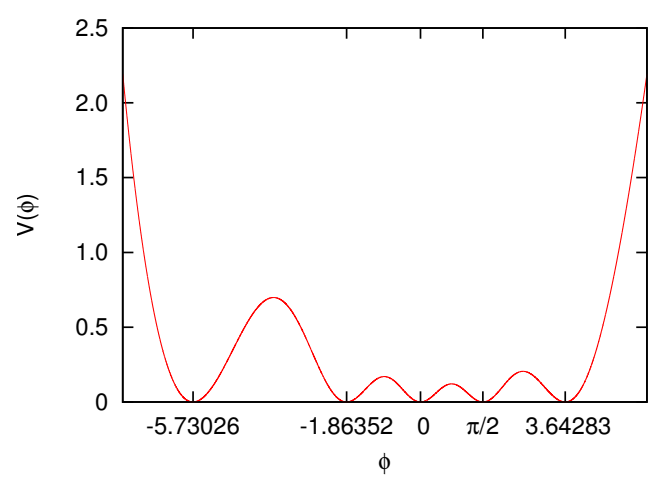

(b)

Figure 13. The modified potential $V(\phi)$ against $\phi$ for $\epsilon=0.05$ and (a) $\gamma=0$, (b) $\gamma=1$.

In the case $\epsilon=0$ the parameter $\gamma$ becomes irrelevant and the potential (4.5) returns to the unperturbed sine-Gordon potential and $\phi=\psi$. For $\epsilon \neq 0$ and $\gamma=0$ the model has the symmetry $\phi=-\phi$, while for $\epsilon, \gamma \neq 0$ there is no symmetry. This can be seen in figure 13 where we plot the potential as a function of $\phi$ for $\epsilon=0.05$ and $\gamma=0$ and $\gamma=1$. By varying the parameters $\epsilon$ and $\gamma$ the effects of this symmetry on the theory can be seen. Note that the topological charge of $\phi(\psi)$, for $\psi$ given by (4.2), is conserved for any value of $\epsilon$ and $\gamma$.

In a similar manner to the NLS case the modified sine-Gordon posses a set of anomalous conservation laws derived in [3] and given by:

$\frac{d \tilde{Q}^{(2 n+1)}}{d t}=\tilde{\beta}^{2 n+1} ; \quad$ with $\tilde{Q}^{(2 n+1)}=\int_{-\infty}^{\infty} d x \tilde{a}_{x}^{(2 n+1)} ; \quad$ where $\tilde{\beta}^{(2 n+1)}=\int_{-\infty}^{\infty} d x \tilde{X} \tilde{\alpha}^{(2 n+1)}$

for $n=0,1,2,3, \ldots$ and

$$
\tilde{X}=\frac{i w}{2} \partial_{-} \phi\left[\frac{d^{2} V}{d \phi^{2}}+16 V-1\right]
$$

which vanishes for the sine-Gordon potential.

If the field configuration transforms under the parity defined in (1.1) as

$$
P(\phi)=-\phi+\text { const. }
$$

and if the potential evaluated on such a solution is even under the parity, i.e.

$$
P(V)=V,
$$

then we have an infinite set of conserved quantities which are conserved asymptotically, i.e.

$$
Q^{(2 n+1)}(t=+\infty)=Q^{(2 n+1)}(t=-\infty) .
$$

This modified model, when $\epsilon=0$, becomes the sine-Gordon model, therefore the system is integrable and has an infinite number of conserved quantities. When $\epsilon \neq 0$ and $\gamma=0$ then the field configuration and potential transform under the parity described in (1.1) 
as in (4.8) and (4.9); therefore the system is quasi-integrable and possesses an infinite number of asymptotically conserved charges. When $\epsilon \neq 0$ and $\gamma \neq 0$ then the symmetries necessary for quasi-integrability are not present; the system is non-integrable and there are no constraints on the charges.

\subsection{The two-soliton configuration for modified sine-Gordon}

As in the NLS case we construct an appropriate two-soliton ansatz for the sine-Gordon in the collective coordinate approximation by patching together two one-kink solutions. We do this in the following way:

$$
\tan (\psi)=e^{(x-a)}-e^{-(x+a)}=2 \sinh (x) e^{-a}
$$

where $a$ is our collective coordinate. When $a$ is large (4.11) represents two well separated kinks; one placed at $-a$ whose field varies between $\left(-\frac{\pi}{2}, 0\right)$ and one placed at $a$ which varies between $\left(0, \frac{\pi}{2}\right)$. For energetic reasons it must be that $a>0$ for all times. This ansatz was used in [12] to test the collective coordinate approximation for the scattering of sine-Gordon kinks and was found to work remarkably well so our ansatz for our modified sine-Gordon model will be based on a generalisation of this ansatz.

To construct a modified approximation ansatz we perform the change of variable as in (4.3), for $\psi$ given by (4.11), to get

$$
\begin{array}{ll}
\phi=\frac{\psi^{2} \epsilon \gamma+\sqrt{\psi^{2} c^{2}+\psi^{4} \epsilon\left(-1+\gamma^{2} \epsilon\right)}}{\psi^{2} \epsilon-c^{2}} & \text { for } x<0, \\
\phi=\frac{\psi^{2} \epsilon \gamma-\sqrt{\psi^{2} c^{2}+\psi^{4} \epsilon\left(-1+\gamma^{2} \epsilon\right)}}{\psi^{2} \epsilon-c^{2}} & \text { for } x>0,
\end{array}
$$

and take this as our two soliton ansatz for the Euler-Lagrange equation associated to (4.1) with the potential given by (4.5). This ansatz returns to the ansatz for the unmodified sine-Gordon in the case $\epsilon=0$. For $\epsilon \neq 0, \gamma=0$ the kinks are altered but the potential retains the symmetry $V(\phi)=V(-\phi)$, whereas for $\epsilon \neq 0, \gamma \neq 0$ this symmetry is lost due to the shift in the vacua which can be seen in figure 13(b).

\subsection{Implementing the approximation in modified sine-Gordon}

We substitute our approximation ansatz (4.12) into the Lagrangian (4.1) (with the change of variable (4.3) and modified potential (4.5)) to find our effective Lagrangian:

$$
L=\frac{g(a)}{2} \dot{a}^{2}-V(a)
$$

where the dot refers to a differentiation with respect to time. The expression for $g(a)$ is given by

$$
g(a)=4 e^{2 a} c^{4} \int_{\infty}^{\infty} d x \frac{A(x, a)}{B(x, a)}
$$


where

$$
\begin{aligned}
A(x, a)= & \sinh ^{2}(x)\left(\epsilon^{2} \tan ^{-1}\left(2 e^{-a} \sinh (x)\right)^{4}\left(\alpha\left(8 \gamma^{2} \epsilon\left(\gamma^{2} \epsilon-1\right)+1\right)+4 \gamma c^{2}\left(2-3 \gamma^{2} \epsilon\right)\right)\right. \\
& -2 c^{2} \epsilon \tan ^{-1}\left(2 e^{-a} \sinh (x)\right)^{2}\left(\alpha\left(1-4 \gamma^{2} \epsilon\right)+2 \gamma c^{2}\right) \\
& \left.-4 \gamma \epsilon^{3}\left(\gamma^{2} \epsilon\left(2 \gamma^{2} \epsilon-3\right)+1\right) \tan ^{-1}\left(2 e^{-a} \sinh (x)\right)^{6}+\alpha c^{4}\right) \\
B(x, a)= & \alpha\left(e^{2 a}+4 \sinh ^{2}(x)\right)^{2}\left(c^{2}-\epsilon \tan ^{-1}\left(2 e^{-a} \sinh (x)\right)^{2}\right)^{4} \\
& \times\left(\epsilon\left(\gamma^{2} \epsilon-1\right) \tan ^{-1}\left(2 e^{-a} \sinh (x)\right)^{2}+c^{2}\right)
\end{aligned}
$$

Moreover, $V(a)$ is:

$$
V(a)=2 e^{2 a} c^{4} \int_{\infty}^{\infty} d x \frac{C(x, a)}{D(x, a)}
$$

where $C(x, a)$ and $D(x, a)$ are given by:

$$
\begin{aligned}
C(x, a)= & \cosh (2 x)\left(\epsilon \left(\epsilon \operatorname { t a n } ^ { - 1 } ( 2 e ^ { - a } \operatorname { s i n h } ( x ) ) ^ { 4 } \left(c^{2}\left(12 \gamma^{2} \epsilon\left(4 \gamma^{2} \epsilon-3\right)+3\right)\right.\right.\right. \\
& \left.-2 \alpha \gamma \epsilon\left(4 \gamma^{2} \epsilon-3\right)\left(4 \gamma^{2} \epsilon-1\right)\right)+c^{2} \tan ^{-1}\left(2 e^{-a} \sinh (x)\right)^{2}\left(4 \alpha \gamma \epsilon\left(3-8 \gamma^{2} \epsilon\right)\right. \\
& \left.+3 c^{2}\left(6 \gamma^{2} \epsilon-1\right)\right)+\epsilon^{2}\left(2 \gamma^{2} \epsilon-1\right)\left(16 \gamma^{2} \epsilon\left(\gamma^{2} \epsilon-1\right)+1\right) \tan ^{-1}\left(2 e^{-a} \sinh (x)\right)^{6} \\
& \left.\left.-6 \alpha c^{4} \gamma\right)+c^{6}\right), \\
D(x, a)= & \left(e^{2 a}+4 \sinh ^{2}(x)\right)^{2}\left(c^{2}-\epsilon \tan ^{-1}\left(2 e^{-a} \sinh (x)\right)^{2}\right)^{4}(-\alpha \gamma \epsilon \\
& \left.+\epsilon\left(\gamma^{2} \epsilon-1\right) \tan ^{-1}\left(2 e^{-a} \sinh (x)\right)^{2}+c^{2}\right)^{2} .
\end{aligned}
$$

For the clarity of the expressions we have introduced and defined $\alpha$ to be:

$$
\alpha \equiv \sqrt{c^{2} \tan ^{-1}\left(2 e^{-a} \sinh (x)\right)^{2}+\epsilon\left(\gamma^{2} \epsilon-1\right) \tan ^{-1}\left(2 e^{-a} \sinh (x)\right)^{4}} .
$$

When $\epsilon=0$ and $\gamma=0$ the expressions for $g(a), V(a)$ revert to those given in [12].

From the Lagrangian 4.13 we derive the equation of motion

$$
g \ddot{a}+\frac{1}{2} \frac{d g}{d a} \dot{a}^{2}+\frac{d V}{d a}=0,
$$

which we solve using the 4th order Runge-Kutta method.

\subsection{Results for sine-Gordon}

First we analyse the scattering of our two kinks for the case $\epsilon=0$ which corresponds to the integrable sine-Gordon model. We compare the trajectories of the kinks as determined using the collective coordinate approximation and using the full numerical simulation for a range of initial velocities $v=\dot{a}(0)$ in order to determine the effective range of validity of our choice of approximation ansatz.

In figure 14 we compare the trajectories of the kinks initially placed at $a=10$ and with initial approach velocities of $v=0.3$ and $v=0.6$. We see that in the integrable system 


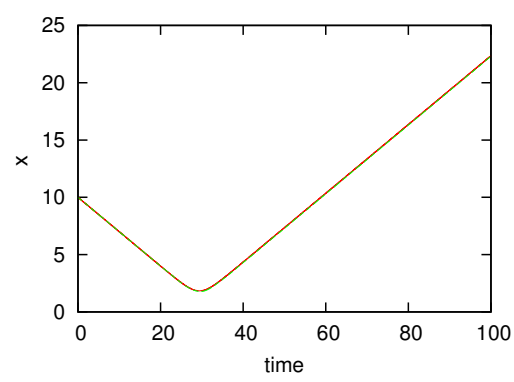

(a)

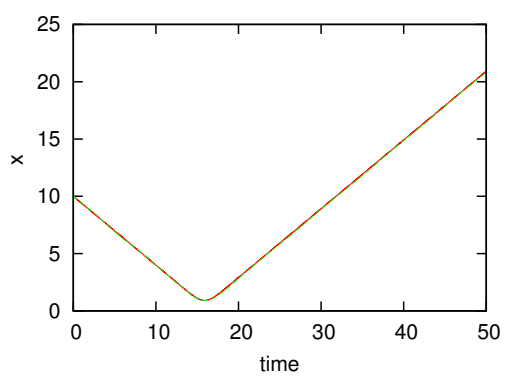

(b)

Figure 14. The distance of a soliton from the centre of mass of a system as a function of time. The system consists of two solitons initially with $a=10$, with an initial velocity towards the centre of mass of (a) $v=0.3$ and (b) $v=0.6$. For each plot the solid line is result of the collective coordinate approximation and the dashed line is the result of the full simulation (these may be indistinguishable).

the collective coordinate approximation with our choice of ansatz gives excellent qualitative agreement with the full numerical simulation up to a high velocity, with percentage difference in soliton positions after collision of $0.21 \%$ for $v=0.3$ at $t=100$ and $0.44 \%$ for $v=0.6$ at $t=50$. This gives us confidence in our modified approximation ansatz as applied to our modified model.

\subsection{Results for modified sine-Gordon}

Now we consider the scattering of solitons when the system is no longer integrable, i.e. for $\epsilon \neq 0$, and analyse the scattering of the two kinks for various values of the parameters $\epsilon$ and $\gamma$. For each set of values we compare the trajectories of the solitons calculated using the collective coordinate approximation with those using the full numerical simulation, and in each simulation we take the initial positions of the solitons corresponding to $a(0)=10$. In the collective coordinate approximation the positions of the kinks are equivalent to $\pm a$ when $\epsilon=0$, but when $\epsilon \neq 0$ the two are no longer equivalent and the location is taken to be the position of the maximum of the energy peak.

In figure 15 we present a series of plots of trajectories for solitons sent together with an initial velocity of $v=0.3$ for different values of $\epsilon$ and $\gamma$. From these plots we can see that the two approaches show excellent agreement when the symmetry necessary for quasi-integrability is present, i.e. $\epsilon \neq 0$ and $\gamma=0$, and the percentage difference in soliton positions at $t=100$ is $0.21 \%$ for $\epsilon=-0.2,0.43 \%$ for $\epsilon=0.4$, and $0.78 \%$ for $\epsilon=1$. However, when the system moves away from quasi-integrability, i.e. $\epsilon \neq 0$ and $\gamma \neq 0$, the two methods show good agreement as the solitons approach each other but the solitons scatter at slightly different distances and with different velocities (though these differences are fairly small). This suggests that quasi-integrability is a sufficient condition for the collective coordinate approximation to accurately model trajectories of kinks in modified sine-Gordon systems. 


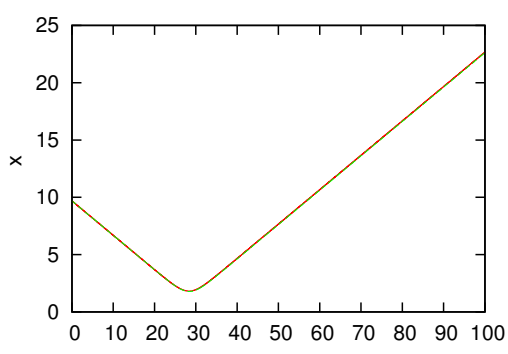

(a)

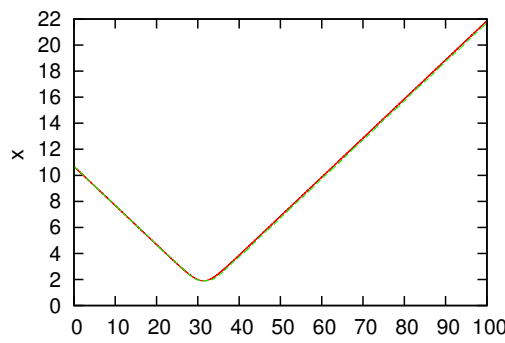

(c)

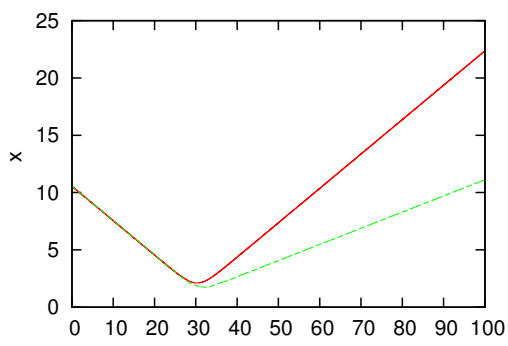

(e)

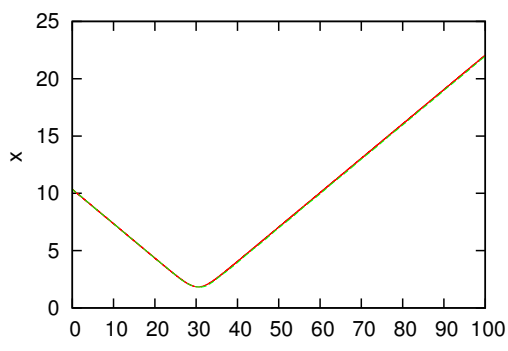

(b)

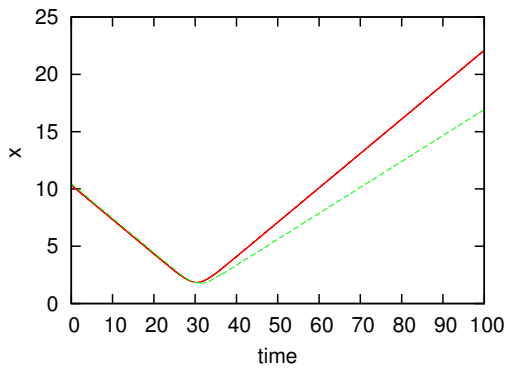

(d)

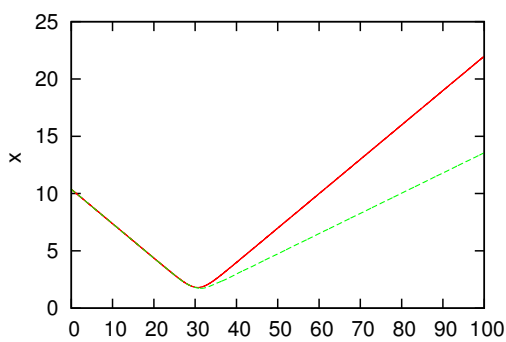

(f)

Figure 15. The distance of a soliton from the centre of mass of a system as a function of time. The system consists of two solitons initially with $a=10$, each with an initial velocity of 0.3 towards the centre of mass. Initial parameter are (a) $\epsilon=-0.2, \gamma=0$; (b) $\epsilon=0.4, \gamma=0$; (c) $\epsilon=1, \gamma=0$; (d) $\epsilon=0.4, \gamma=0.1$; (e) $\epsilon=0.4, \gamma=0.2$; and (f) $\epsilon=0.4, \gamma=-0.2$. For each plot the solid line is result of the collective coordinate approximation and the dashed line is the result of the full simulation (for (a), (b) and (c) these are indistinguishable).

We also consider the quasi-conservation of the first non-trivial charge beyond the energy itself, namely, $\tilde{Q}^{(4)}(t)$ defined in (4.6) by calculating both the anomaly $\tilde{\beta}^{(3)}$ and the time integrated anomaly which is given by:

$$
\tilde{\chi}^{(3)}=-\frac{1}{2} \int_{t_{0}}^{t} d t^{\prime} \tilde{\beta}^{(3)}=4 \int_{t_{0}}^{t} d t^{\prime} \int_{-\infty}^{\infty} d x \partial_{-} \phi \partial_{-}^{2} \phi\left[\frac{d^{2} V}{d \phi^{2}}+16 V-1\right],
$$

where $\partial_{-}=\partial_{t}-\partial_{x}$ and $t_{0}$ is the initial time of the simulation which is usually taken to be zero. 


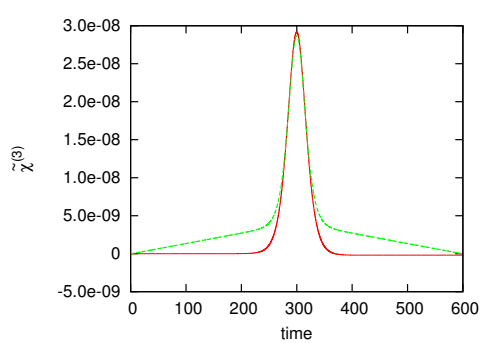

(a)

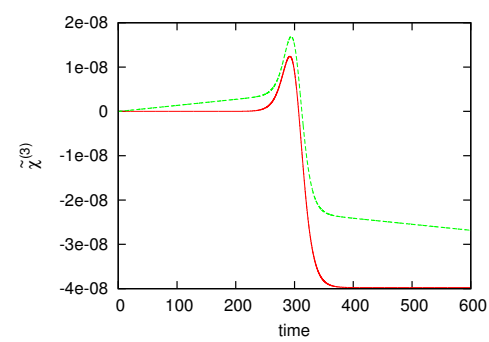

(b)

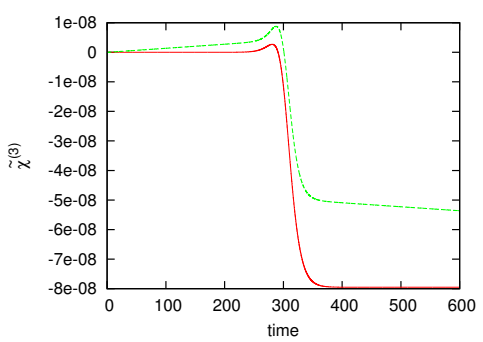

(c)

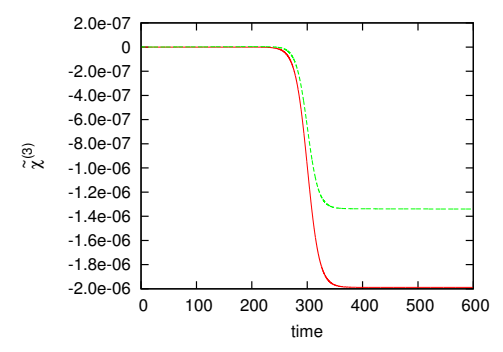

(d)

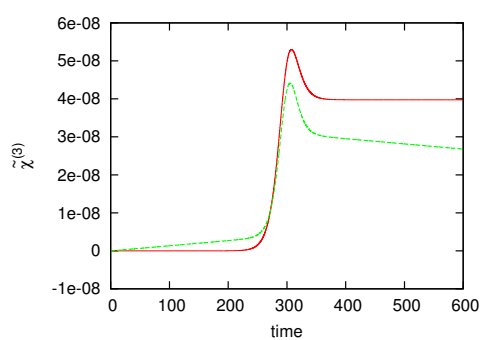

(e)

Figure 16. The time-integrated anomaly for solitons initially with $a=20$ with velocity 0.05 towards the centre of mass and $\epsilon=0.000001$. $\gamma$ is chosen to be (a) $\gamma=0.00001$, (b) $\gamma=0.002$, (c) $\gamma=0.004$, (d) $\gamma=0.1$ and (e) $\gamma=-0.002$. The solid lines are the results for the collective coordinate approximation and the dashed lines are results for the full simulation.

Figure 16 is the plot of the time-integrated anomaly as a function of time for solitons placed at $a=20$ with initial velocity $v=0.05$, with $\epsilon=0.000001$ and various values of $\gamma$. Notice that in the full simulation the time-integrated anomaly is always slowly increasing prior to the scattering of the solitons and slowly decreasing after the scattering; this is due to slight fluctuations away from zero in the anomaly which by itself is probably a result of numerical errors rather than any physical effect. This error increases as $\epsilon$ increases and so it becomes difficult to compare the results, this is why we present plots only for a small value of $\epsilon$. We see that when $\gamma$ is small the collective coordinate approximation and the full simulation are in excellent agreement, and far away from the scattering the time-integrated anomaly is close to zero, as expected, when $\gamma$ is small and the model is close to the symmetry described in (4.8) and (4.9). When $\gamma$ is taken further from zero we move from a model with approximate symmetry to a model where this symmetry is broken. This is confirmed by our results as seen in figure 16 which show that the further $\gamma$ is from zero the further the time-integrated anomaly is from zero after the scattering of the solitons. Moreover, the figures 16(b) and 16(e) show that the symmetry can be broken in either direction depending on the sign of $\gamma$. The collective coordinate approximation still gives a good qualitative approximation to the behaviour of the time-integrated anomaly as we move away from the symmetric case though the values, as to be expected, are not exactly the same as seen in full simulations. These observations have been checked for several values of $\epsilon$. 


\section{Conclusions}

In this paper we have considered the applicability of the collective coordinate approximation to the description of the scattering of solitons in models which are deformations of the NLS or the sine-Gordon model. The deformation of these models changes their integrability properties, either completely or partially (the models become 'quasi-integrable'). Hence we have considered a modified NLS and a modified sine-Gordon system for which the trajectories were already known from full simulations studied in [2] and [3]. Moreover, [2] and [3] have also suggested that quasi-integrability could be related to a particular symmetry of the field configurations (for configurations possessing the necessary symmetry the anomaly terms could vanish and so lead to quasi-integrability), so we looked at these properties using the collective coordinate approximation.

In the modified NLS the approximation works very well in the majority of cases and for a good range of initial conditions with a well chosen approximation ansatz. The predominant influence on the accuracy of the approximation is the time the two solitons spend in close proximity of each other during their interaction; and for simulations where the solitons do not come closer together than the width of one soliton the collective coordinate approximation accurately reproduces the scattering of the solitons and their anomaly even for values of initial velocity up to $v=0.2$. In these cases the trajectories, heights and phase difference of the solitons during their scattering, calculated using the collective coordinate approximation, are often indistinguishable from the those calculated using a full numerical method. Moving the system away from integrability, i.e. increasing the deformation parameter $\epsilon$, reduces the accuracy only slightly when the solitons stay far enough apart during their scattering and the results are very good in the range of $\epsilon$ that we investigated, namely $|\varepsilon|<0.9$. For the vast majority of initial conditions the solitons maintain enough distance from each other during their interaction to ensure the accuracy of the approximation, and even show good qualitative agreement for the time-integrated anomalies.

When the solitons come closer together than the width of one soliton during their interaction the general behaviour of the solitons (trajectory, height and anomaly) is still reproduced but the accuracy of the approximation is reduced. We think this is probably because in the full simulation the solitons deform one another away from the form given by (3.15) when they are in close proximity, and when $\epsilon \neq 0$ they also radiate out energy, and the collective coordinate approximation does not allow this to happen. This effect is exacerbated as the system moves away from integrability because this radiation/deformation effect increases with increasing $\epsilon$.

The effect of quasi-integrability in the modified NLS is difficult to assess fully, as the collective coordinate approximation is incredibly reliable in the quasi-integrable case i.e. for the initial phase difference of $\delta=n \pi$ where $n$ are odd integers. However, for the remaining initial values of the phase difference where the system is quasi-integrable $(\delta=n \pi$ for $n$ even integers) the effect of the proximity of the solitons (as discussed above) eclipses any effect of quasi-integrability.

In the modified sine-Gordon case the approximation very accurately describes the trajectories and anomalies of scattering kinks when the system is either integrable or quasi- 
integrable (i.e. $\gamma=0$ ) up to initial velocities of $v=0.6$ and for values of up to $\epsilon=1$. However, when the field configuration moves away from the symmetry necessary for quasiintegrability (i.e. when $\gamma$ moves away from 0 ) the collective coordinate approximation becomes less accurate for both the trajectories and the anomalies.

These observations suggest that the collective coordinate approximation is a very useful tool to study various properties of the scatterings of solitons, topological or not, and so can be used also to investigate quasi-integrability in other perturbations of integrable models. In modified sine-Gordon models the presence of the symmetries necessary for quasi-integrability seem to be a sufficient condition to ensure accuracy, but in any model care should be taken if the solitons have the opportunity to strongly deform each other.

\section{Acknowledgments}

HB is supported by an STFC studentship. We wish to thank L. A. Ferreira and G. Luchini for helpful conversations.

\section{A Expressions for $a_{x}^{(3,-n)}$ and $\alpha^{(3,-n)}$ for $n=0,1,2,3,4$}

The components $a_{x}^{(3,-n)}$ introduced in (3.6), as calculated in [2], (in terms of the fields $R$ and $\varphi$ as defined in (3.11)) are:

$$
\begin{aligned}
a_{x}^{(3,0)} & =\frac{i}{2} \partial_{x} \varphi \\
a_{x}^{(3,-1)} & =2 i \eta R \\
a_{x}^{(3,-2)} & =i \eta \partial_{x} \varphi R \\
a_{x}^{(3,-3)} & =\frac{i}{2 R}\left(4 \eta^{2} R^{3}+\eta\left(\partial_{x} \varphi\right)^{2} R^{2}-2 \eta \partial_{x}^{2} R R+\eta\left(\partial_{x} R\right)^{2}\right), \\
a_{x}^{(3,-4)} & =\frac{i}{4 R}\left(12 \eta^{2} \partial_{x} \varphi R^{3}-6 \eta R\left(\partial_{x}^{2} \varphi \partial_{x} R+\partial_{x} \varphi \partial_{x}^{2} R\right)+3 \eta \partial_{x} \varphi\left(\partial_{x} R\right)^{2}\right. \\
& \left.\quad+\eta\left(\left(\partial_{x} \varphi\right)^{3}-4 \partial_{x}^{3} \varphi\right) R^{2}\right) .
\end{aligned}
$$

The components $\alpha^{(3,-n)}$ introduced in (3.6) (in terms of the fields $R$ and $\varphi$ as defined in $(3.11))$ are:

$$
\begin{aligned}
\alpha^{(3,0)} & =1 \\
\alpha^{(3,-1)} & =0 \\
\alpha^{(3,-2)} & =2 \eta R \\
\alpha^{(3,-3)} & =2 \eta \partial_{x} \varphi R \\
\alpha^{(3,-4)} & =6 \eta^{2} R^{2}+\frac{3}{2} \eta\left(\partial_{x} \varphi\right)^{2} R-2 \eta \partial_{x}^{2} R+\frac{3 \eta\left(\partial_{x} R\right)^{2}}{2 R} .
\end{aligned}
$$




\section{B Component functions for the effective Lagrangian of the modified NLS}

For convenience we have defined $\omega_{i}=(1+\epsilon) a_{i}(t)\left(x+\xi_{i}(t)\right)$ for $i=1,2$.

$$
\begin{aligned}
& I_{\xi_{1}}=\int_{-\infty}^{\infty} d x \frac{1}{4}\left(\frac{a_{1} \sqrt{\epsilon+2}}{\cosh \left(\omega_{1}\right)}\right)^{\frac{1}{\epsilon+1}}\left(4 a_{1} \sin \left(\theta_{1}-\theta_{2}\right) \tanh \left(\omega_{1}\right)\left(\frac{a_{2} \sqrt{\epsilon+2}}{\cosh \left(\omega_{2}\right)}\right)^{\frac{1}{\epsilon+1}}\right. \\
& \left.+\mu_{1}\left(\left(\frac{a_{1} \sqrt{\epsilon+2}}{\cosh \left(\omega_{1}\right)}\right)^{\frac{1}{\epsilon+1}}+\cos \left(\theta_{1}-\theta_{2}\right)\left(\frac{a_{2} \sqrt{\epsilon+2}}{\cosh \left(\omega_{2}\right)}\right)^{\frac{1}{\epsilon+1}}\right)\right) \\
& I_{\xi_{2}}=\int_{-\infty}^{\infty} d x \frac{1}{4}\left(\frac{a_{2} \sqrt{\epsilon+2}}{\cosh \left(\omega_{2}\right)}\right)^{\frac{1}{\epsilon+1}}\left(-4 a_{2} \sin \left(\theta_{1}-\theta_{2}\right) \tanh \left(\omega_{2}\right)\left(\frac{a_{1} \sqrt{\epsilon+2}}{\cosh \left(\omega_{1}\right)}\right)^{\frac{1}{\epsilon+1}}\right. \\
& \left.+\mu_{2}\left(\cos \left(\theta_{1}-\theta_{2}\right)\left(\frac{a_{1} \sqrt{\epsilon+2}}{\cosh \left(\omega_{1}\right)}\right)^{\frac{1}{\epsilon+1}}+\left(\frac{a_{2} \sqrt{\epsilon+2}}{\cosh \left(\omega_{2}\right)}\right)^{\frac{1}{\epsilon+1}}\right)\right) \\
& I_{\mu_{1}}=\int_{-\infty}^{\infty} d x \frac{\left(\xi_{1}+2 x\right)}{4}\left(\frac{a_{1} \sqrt{\epsilon+2}}{\cosh \left(\omega_{1}\right)}\right)^{\frac{1}{\epsilon+1}}\left(\left(\frac{a_{1} \sqrt{\epsilon+2}}{\cosh \left(\omega_{1}\right)}\right)^{\frac{1}{\epsilon+1}}\right. \\
& \left.+\cos \left(\theta_{1}-\theta_{2}\right)\left(\frac{a_{2} \sqrt{\epsilon+2}}{\cosh \left(\omega_{2}\right)}\right)^{\frac{1}{\epsilon+1}}\right) \\
& I_{\mu_{2}}=\int_{-\infty}^{\infty} d x \frac{\left(\xi_{2}+2 x\right)}{4}\left(\frac{a_{2} \sqrt{\epsilon+2}}{\cosh \left(\omega_{2}\right)}\right)^{\frac{1}{\epsilon+1}}\left(\cos \left(\theta_{1}-\theta_{2}\right)\left(\frac{a_{1} \sqrt{\epsilon+2}}{\cosh \left(\omega_{1}\right)}\right)^{\frac{1}{\epsilon+1}}\right. \\
& \left.+\left(\frac{a_{2} \sqrt{\epsilon+2}}{\cosh \left(\omega_{2}\right)}\right)^{\frac{1}{\epsilon+1}}\right) \\
& I_{a_{1}}=\int_{-\infty}^{\infty} d x \frac{1}{a_{1}}\left(\frac{a_{1} \sqrt{\epsilon+2}}{\cosh \left(\omega_{1}\right)}\right)^{\frac{1}{\epsilon+1}}\left(-2 a_{1}^{2} t\left(\left(\frac{a_{1} \sqrt{\epsilon+2}}{\cosh \left(\omega_{1}\right)}\right)^{\frac{1}{\epsilon+1}}+\cos \left(\theta_{1}-\theta_{2}\right)\left(\frac{a_{2} \sqrt{\epsilon+2}}{\cosh \left(\omega_{2}\right)}\right)^{\frac{1}{\epsilon+1}}\right)\right. \\
& \left.+\sin \left(\theta_{1}-\theta_{2}\right)\left(a_{1}\left(\xi_{1}+x\right) \tanh \left(\omega_{1}\right)-\frac{1}{\epsilon+1}\right)\left(\frac{a_{2} \sqrt{\epsilon+2}}{\cosh \left(\omega_{2}\right)}\right)^{\frac{1}{\epsilon+1}}\right) \\
& I_{a_{2}}=\int_{-\infty}^{\infty} d x \frac{1}{a_{2}}\left(\frac{a_{2} \sqrt{\epsilon+2}}{\cosh \left(\omega_{2}\right)}\right)^{\frac{1}{\epsilon+1}}\left(-2 a_{2}^{2} t\left(\cos \left(\theta_{1}-\theta_{2}\right)\left(\frac{a_{1} \sqrt{\epsilon+2}}{\cosh \left(\omega_{1}\right)}\right)^{\frac{1}{\epsilon+1}}+\left(\frac{a_{2} \sqrt{\epsilon+2}}{\cosh \left(\omega_{2}\right)}\right)^{\frac{1}{\epsilon+1}}\right)\right. \\
& \left.+\sin \left(\theta_{1}-\theta_{2}\right)\left(-a_{2}\left(\xi_{2}+x\right) \tanh \left(\omega_{2}\right)+\frac{1}{\epsilon+1}\right)\left(\frac{a_{1} \sqrt{\epsilon+2}}{\cosh \left(\omega_{1}\right)}\right)^{\frac{1}{\epsilon+1}}\right) \\
& I_{\lambda_{1}}=-\int_{-\infty}^{\infty} d x\left(\frac{a_{1} \sqrt{\epsilon+2}}{\cosh \left(\omega_{1}\right)}\right)^{\frac{1}{\epsilon+1}}\left(\left(\frac{a_{1} \sqrt{\epsilon+2}}{\cosh \left(\omega_{1}\right)}\right)^{\frac{1}{\epsilon+1}}+\cos \left(\theta_{1}-\theta_{2}\right)\left(\frac{a_{2} \sqrt{\epsilon+2}}{\cosh \left(\omega_{2}\right)}\right)^{\frac{1}{\epsilon+1}}\right) \\
& I_{\lambda_{2}}=-\int_{-\infty}^{\infty} d x\left(\frac{a_{2} \sqrt{\epsilon+2}}{\cosh \left(\omega_{2}\right)}\right)^{\frac{1}{\epsilon+1}}\left(\cos \left(\theta_{1}-\theta_{2}\right)\left(\frac{a_{1} \sqrt{\epsilon+2}}{\cosh \left(\omega_{1}\right)}\right)^{\frac{1}{\epsilon+1}}+\left(\frac{a_{2} \sqrt{\epsilon+2}}{\cosh \left(\omega_{2}\right)}\right)^{\frac{1}{\epsilon+1}}\right)
\end{aligned}
$$




$$
\begin{aligned}
V= & \int_{-\infty}^{\infty} d x\left(a_{1}^{2}\left(\frac{a_{1}}{\cosh \left(\omega_{1}\right)}\right)^{\frac{2}{\epsilon+1}}\left(1+\tanh ^{2}\left(\omega_{1}\right)\right)+a_{2}^{2}\left(\frac{a_{2}}{\cosh \left(\omega_{2}\right)}\right)^{\frac{2}{\epsilon+1}}\left(1+\tanh ^{2}\left(\omega_{2}\right)\right)\right. \\
& +\left(a_{1}^{2}+a_{2}^{2}+2 a_{1} a_{2} \tanh \left(\omega_{1}\right) \tanh \left(\omega_{2}\right)\right) \cos \left(\theta_{1}-\theta_{2}\right)\left(\frac{a_{1} a_{2}}{\cosh \left(\omega_{1}\right) \cosh \left(\omega_{2}\right)}\right)^{\frac{1}{\epsilon+1}} \\
& -\frac{2}{2+\epsilon}\left(\left(\frac{a_{1}}{\cosh \left(\omega_{1}\right)}\right)^{\frac{2}{\epsilon+1}}+\left(\frac{a_{2}}{\cosh \left(\omega_{2}\right)}\right)^{\frac{2}{\epsilon+1}}+2 \cos \left(\theta_{1}-\theta_{2}\right)\left(\frac{a_{1} a_{2}}{\cosh \left(\omega_{1}\right) \cosh \left(\omega_{2}\right)}\right)^{\frac{1}{\epsilon+1}}\right)^{2+\epsilon} \\
& \left.+\frac{1}{4}\left(\mu_{1}^{2}\left(\frac{a_{1}}{\cosh \left(\omega_{1}\right)}\right)^{\frac{2}{\epsilon+1}}+\mu_{2}^{2}\left(\frac{a_{2}}{\cosh \left(\omega_{2}\right)}\right)^{\frac{2}{\epsilon+1}}\right)\right)
\end{aligned}
$$

Open Access. This article is distributed under the terms of the Creative Commons Attribution License (CC-BY 4.0), which permits any use, distribution and reproduction in any medium, provided the original author(s) and source are credited.

\section{References}

[1] L.A. Ferreira and W.J. Zakrzewski, The concept of quasi-integrability: a concrete example, JHEP 05 (2011) 130 [arXiv: 1011.2176] [INSPIRE].

[2] L.A. Ferreira, G. Luchini and W.J. Zakrzewski, The concept of quasi-integrability for modified non-linear Schrödinger models, JHEP 09 (2012) 103 [arXiv:1206.5808] [INSPIRE].

[3] L.A. Ferreira and W.J. Zakrzewski, Numerical and analytical tests of quasi-integrability in modified sine-Gordon models, JHEP 01 (2014) 058 [arXiv:1308.4412] [INSPIRE].

[4] A. Sánchez and A.R. Bishop, Collective Coordinates and Length-Scale Competition in Spatially Inhomogeneous Soliton-Bearing Equations, SIAM Rev. 40 (1998) 579.

[5] H.E. Baron, G. Luchini and W.J. Zakrzewski, Collective coordinate approximation to the scattering of solitons in the $(1+1)$ dimensional NLS model, J. Phys. A 47 (2014) 265201 [arXiv: 1308.4072] [INSPIRE].

[6] A.A. Thiele, Steady-State Motion of Magnetic Domains, Phys. Rev. Lett. 30 (1973) 230.

[7] O.A. Tretiakov, D. Clarke, Gia-Wei Chern, Ya.B. Bazaliy and O. Tchernyshyov, Dynamics of Domain Walls in Magnetic Nanostrips, Phys. Rev. Lett. 100 (2008) 127204.

[8] N.R. Quintero, F.G. Mertens and A.R. Bishop, Generalized traveling-wave method, variational approach, and modified conserved quantities for the perturbed nonlinear Schrödinger equation, Phys. Rev. E 82 (2010) 016606.

[9] N.S. Manton and P.M. Sutcliffe, Topological Solitons, Cambridge University Press (2004).

[10] N.S. Manton, An Effective Lagrangian for Solitons, Nucl. Phys. B 150 (1979) 397 [InSPIRE].

[11] J.H.P. Dawes and H. Susanto, Variational approximation and the use of collective coordinates, Phys. Rev. E 87 (2013) 063202.

[12] P.M. Sutcliffe, Classical and quantum kink scattering, Nucl. Phys. B 393 (1993) 211 [INSPIRE].

[13] A. Dar, M. Kugler, Y. Dothan and S. Nussinov, Diffraction patterns for elastic scattering of elementary particles, Phys. Lett. 11 (1964) 265 [INSPIRE]. 